\title{
STAR FORMATION SUPPRESSION IN COMPACT GROUP GALAXIES: A NEW PATH TO QUENCHING?
}

\author{
K. Alatalo ${ }^{1,2,22}$, P. N. Appleton ${ }^{1,3}$, U. Lisenfeld ${ }^{4}$, T. Bitsakis ${ }^{3,5}$, L. Lanz ${ }^{1}$, M. Lacy ${ }^{6}$, V. Charmandaris ${ }^{7,8,9}$, M. Cluver ${ }^{10}$, \\ M. A. Dopita ${ }^{11,12,13}$, P. Guillard ${ }^{14,15}$, T. JARretT ${ }^{16}$, L. J. Kewley ${ }^{11}$, K. Nyland ${ }^{17}$, P. M. OGLE ${ }^{1}$, J. Rasmussen ${ }^{18,19}$, \\ J. A. Rich ${ }^{1,2}$, L. Verdes-MonteneGro ${ }^{20}$, C. K. Xu ${ }^{1,3}$, AND M. Yun ${ }^{21}$ \\ ${ }^{1}$ Infrared Processing \& Analysis Center, California Institute of Technology, Pasadena, CA 91125, USA; kalatalo@carnegiescience.edu \\ ${ }^{2}$ Observatories of the Carnegie Institution of Washington, 813 Santa Barbara Street, Pasadena, CA 91101, USA \\ ${ }^{3}$ NASA Herschel Science Center, IPAC, California Institute of Technology, Pasadena, CA 91125, USA \\ ${ }^{4}$ Departamento de Física Teórica y del Cosmos, Universidad de Granada, Granada, Spain \\ ${ }^{5}$ Instituto de Astronomía, Universidad Nacional Autónoma de México, Aptdo. Postal 70-264, 04510, México, D.F., Mexico \\ ${ }^{6}$ National Radio Astronomy Observatory, 520 Edgemont Road, Charlottesville, VA 22903, USA \\ ${ }^{7}$ Institute for Astronomy, Astrophysics, Space Applications \& Remote Sensing, National Observatory of Athens, GR-15236 Penteli, Greece \\ ${ }^{8}$ Department of Physics, University of Crete, GR-71003 Heraklion, Greece \\ ${ }^{9}$ Chercheur Associé, Observatoire de Paris, F-75014 Paris, France \\ ${ }^{10}$ Department of Physics, University of the Western Cape, Robert Sobukwe Road, Bellville, 7535, South Africa \\ ${ }^{11}$ Research School of Astronomy and Astrophysics, Australian National University, Cotter Rd., Weston, ACT 2611, Australia \\ ${ }_{12}$ Astronomy Department, King Abdulaziz University, P.O. Box 80203, Jeddah, Saudi Arabia \\ ${ }^{13}$ Institute for Astronomy, University of Hawaii, 2680 Woodlawn Drive, Honolulu, HI 96822, USA \\ ${ }^{14}$ Sorbonne Universités, UPMC Univ Paris 6, CNRS et, UMR 7095, Institut d'Astrophysique de Paris, 98 bis bd Arago, F-75014 Paris, France \\ ${ }^{16}$ Astrophysics Cosmology and Gravity Centre, Department of Astronomy, University of Cape Town, Private Bag X3, Rondebosch, 7701, South Africa \\ ${ }^{17}$ Netherlands Institute for Radio Astronomy (ASTRON), Postbus 2, 7990 AA Dwingeloo, The Netherlands \\ ${ }^{18}$ Dark Cosmology Centre, Niels Bohr Institute, University of Copenhagen, Juliane Maries Vej 30, DK-2100 Copenhagen, Denmark \\ ${ }_{19}$ Technical University of Denmark, Department of Physics, Building 309, DK-2800 Kgs. Lyngby, Denmark \\ ${ }^{20}$ Departamento Astronomía Extragaláctica, Instituto Astrofísica Andaluća (CSIC), Glorieta de la Astronomia s/n E-18008 Granada, Spain \\ ${ }^{21}$ University of Massachusetts, Astronomy Department, Amherst, MA 01003, USA \\ Received 2015 July 18; accepted 2015 September 18; published 2015 October 14
}

\begin{abstract}
We present $\mathrm{CO}(1-0)$ maps of 12 warm $\mathrm{H}_{2}$-selected Hickson Compact Groups (HCGs), covering 14 individually imaged warm $\mathrm{H}_{2}$ bright galaxies, with the Combined Array for Research in Millimeter Astronomy. We found a variety of molecular gas distributions within the HCGs, including regularly rotating disks, bars, rings, tidal tails, and possibly nuclear outflows, though the molecular gas morphologies are more consistent with spirals and earlytype galaxies than mergers and interacting systems. Our CO-imaged HCG galaxies, when plotted on the Kennicutt-Schmidt relation, shows star formation (SF) suppression of $\langle\mathscr{S}\rangle=10 \pm 5$, distributed bimodally, with five objects exhibiting suppressions of $\mathscr{S} \gtrsim 10$ and depletion timescales $\gtrsim 10 \mathrm{Gyr}$. This SF inefficiency is also seen in the efficiency per freefall time of Krumholz et al. We investigate the gas-to-dust ratios of these galaxies to determine if an incorrect $\mathrm{L}_{\mathrm{CO}}-M\left(\mathrm{H}_{2}\right)$ conversion caused the apparent suppression and find that HCGs have normal gas-to-dust ratios. It is likely that the cause of the apparent suppression in these objects is associated with shocks injecting turbulence into the molecular gas, supported by the fact that the required turbulent injection luminosity is consistent with the bright $\mathrm{H}_{2}$ luminosity reported by Cluver et al. Galaxies with high SF suppression $(\mathscr{S} \gtrsim 10)$ also appear to be those in the most advanced stages of transition across both optical and infrared color space. This supports the idea that at least some galaxies in HCGs are transitioning objects, where a disruption of the existing molecular gas in the system suppresses SF by inhibiting the molecular gas from collapsing and forming stars efficiently. These observations, combined with recent work on poststarburst galaxies with molecular reservoirs, indicates that galaxies do not need to expel their molecular reservoirs prior to quenching SF and transitioning from blue spirals to red early-type galaxies. This may imply that SF quenching can occur without the need to starve a galaxy of cold gas first.
\end{abstract}

Key words: galaxies: evolution - galaxies: kinematics and dynamics - galaxies: star formation

\section{INTRODUCTION}

The present-day galaxy population has a bimodal distribution, comprised of blue spiral galaxies and red elliptical and lenticular galaxies (Tinsley 1978; Strateva et al. 2001; Baldry et al. 2004), with a dearth of galaxies at intermediate optical colors (Bell et al. 2003). Their rarity suggests that galaxies transition rapidly in colors. However, Schawinski et al. (2014) showed that selecting transitioning objects based on color leads to an overestimation of morphologically transforming objects, as the majority of green valley objects are not those undergoing

${ }^{22}$ Hubble fellow. the rapid morphological transformation from spiral to elliptical, but were mainly spiral galaxies that were undergoing secular evolution. More recently, Smethurst et al. (2015) supported this picture of spiral galaxies transitioning at intermediate rates in the green valley, but also showed that early type galaxies transition more rapidly.

Many early transitioning scenarios posited that the majority of molecular gas in galaxies is depleted prior to the quenching of star formation (SF; Sanders \& Mirabel 1996; Hopkins et al. 2006), through both supernova and active galactic nucleus (AGN) feedback mechanisms (Springel et al. 2005). However, recent observations have started to question whether the two- 
stage scenario consisting of (1) the expulsion of star-forming gas followed by (2) the cessation of SF is the exclusive evolutionary picture. Studies now show that many poststarburst galaxies contain substantial reservoirs of molecular gas (French et al. 2015; Rowlands et al. 2015) and are dustier than normal galaxies (Yesuf et al. 2014). This shows that removing all starforming material before transitioning is not a requirement in a galaxy's transformation from blue to red.

With the advent of the Wide-field Infrared Survey Explorer (WISE; Wright et al. 2010) mission, evidence has accumulated that mid-infrared (IR) colors can also be used to identify phases of transitioning galaxies (Ko et al. 2013). Alatalo et al. (2014b) showed the existence of a prominent bifurcation between starforming spiral galaxies and quiescent early-type galaxies in the WISE [4.6]-[12] $\mu \mathrm{m}$ colors, deeming this the "Infrared Transition Zone" (IRTZ). Objects within the IRTZ have red optical colors (also described in Ko et al. 2013), suggesting that galaxies traverse the optical green valley before the IRTZ. Assuming that [4.6]-[12] $\mu \mathrm{m}$ WISE colors trace the interstellar medium (ISM) within the galaxy and optical colors trace the mean stellar population age (Donoso et al. 2012), which further supports the idea that a non-negligible number of galaxies quench SF before shedding their ISMs.

Many plausible mechanisms have been introduced to explain this transition, including major mergers (Toomre \& Toomre 1972; Springel et al. 2005); experiencing ram pressure stripping, and strangulation when falling into a galaxy cluster (Bekki et al. 2002; Blanton \& Moustakas 2009; and references therein); morphological quenching (Martig et al. 2009, 2013); minor mergers (Qu et al. 2010; Eliche-Moral et al. 2012; Alatalo et al. 2014c, 2015b); AGN feedback (Hopkins et al. 2006; Feruglio et al. 2010; Fischer et al. 2010; Alatalo et al. 2011; Sturm et al. 2011; Aalto et al. 2012; Cicone et al. 2012, 2014; Alatalo 2015), and tidal disruption and harassment through group interactions (Hickson et al. 1992; Zabludoff \& Mulchaey 1998; Bitsakis et al. 2010, 2014; Walker et al. 2010). Given their low galaxy velocity dispersion and high density, as well as their short-lived nature (Hickson 1982), compact groups serve as an ideal environment in which to study galaxy transformation.

Hickson compact groups (HCGs) are defined as "small, relatively isolated systems of typically four or five galaxies in close proximity to one another" (Hickson 1982, 1997). They tend to have a high fraction of early-type galaxies (E/S0), evidence of tidal interactions, and high density structure with low velocity dispersion (Hickson 1982, 1997) and deficiencies in $\mathrm{H}_{\mathrm{I}}$ compared with isolated galaxies (Verdes-Montenegro et al. 2001; Borthakur et al. 2010). Compact groups appear to go through an evolutionary phase that can be traced by neutral gas depletion (Verdes-Montenegro et al. 2001). In the later stages of depletion, $\mathrm{H}_{\mathrm{I}}$ is found less in the galaxies and more in the intragalactic medium (IGM; Borthakur et al. 2010), with a rise in the fraction of groups containing extended group-wide X-ray emission (Ponman et al. 1996). However, the origin of the extended X-ray emission is still unclear for HCGs in general (see Rasmussen et al. 2008), with strong starburst winds being the partial cause in at least one system (O'Sullivan et al. 2014a, 2014b). The fraction of galaxy types also evolves in concert with the neutral gas depletion, with spiral-rich groups at early times and elliptical-rich groups later in the sequence (Bitsakis et al. 2010, 2011, 2014).
It also appears that the galaxies within the HCG transform rapidly. No Spitzer color bimodality was reported by Lacy et al. (2004, 2007) between early-type and late-type galaxies in a sample of field galaxies, but Johnson et al. (2007) documented a marked Spitzer IR "gap" in compact group galaxies, with very few galaxies observed between the star-forming cloud and the quiescent cloud. Walker et al. (2010) suggested that compact group galaxies within this "gap" were likely in the midst of a rapid morphological transformation. Cluver et al. (2013) showed that the "gap" galaxies in compact groups tend to have warm hydrogen emission traced by the Spitzer Infrared Spectrograph (IRS; Houck et al. 2004) that is enhanced beyond the level that photon-dominated regions powered by SF alone could explain, termed Molecular Hydrogen Emission Galaxies (MOHEGs; Ogle et al. 2007). Cluver et al. (2013) suggested that this enhanced $\mathrm{H}_{2}$ emission might be energized by shocks caused by collisions with the clumpy intragroup medium. MOHEG "gap" HCGs therefore represent ideal laboratories to test the interplay between rapid galaxy transition, SF quenching, and the disruption of the star-forming fuel and ISM.

Evidence that SF might be taking place inefficiently in transitioning galaxies has started to mount, including in radio galaxies (Nesvadba et al. 2010; Guillard et al. 2015), AGN-driven molecular outflow host NGC 1266 (Alatalo et al. 2015b), as well as in HCGs. Both the shock in Stephan's Quintet (Appleton et al. 2006; Guillard et al. 2009, 2012a; Konstantopoulos et al. 2014) and the HCG galaxy HCG 57a (Alatalo et al. 2014a) seem to exhibit suppressed SF. In these cases, the authors suggested that the injection of turbulence might be causing this SF inhibition, either from collisions with the radio jets or AGN outflows, or the collisions and interactions within the group environment.

We present new Combined Array from Research in Millimeter Astronomy (CARMA) ${ }^{23} \mathrm{CO}(1-0)$ observations of 12 HCGs, including multiple galaxies considered "gap" galaxies and MOHEGs (shown in Table 1). In Section 2, we describe the sample selection and observations from CARMA, including reduction and analysis methods. In Section 3, we present the molecular properties of the sample, including their position on the Kennicutt-Schmidt (K-S) relation (Kennicutt 1998). In Section 4, we discuss these results in the context of transitioning galaxies. In Section 5, we summarize our results. We comment and present the maps of individual galaxies in the appendix. The cosmological parameters $H_{0}=70 \mathrm{~km} \mathrm{~s}^{-1}, \Omega_{\mathrm{m}}=0.3$ and $\Omega_{\Lambda}=0.7$ (Spergel et al. 2007) are used throughout.

\section{OBSERVATIONS AND ANALYSIS}

\subsection{Sample Selection}

While single dish observations are able to provide information about the CO luminosity, and possibly some information about the molecular gas kinematics (such as line-width and line-shape), it is unable to provide spatial information about the molecular gas. Interferometry is able to provide information about the extent and distribution of the molecular gas, allowing for direct comparisons between the CO and stellar mass, or SF. For this reason, we chose to use CARMA to follow up a subset of the CO-bright, warm $\mathrm{H}_{2}$-bright $\mathrm{HCG}$ galaxies presented in Lisenfeld et al. (2014).

\footnotetext{
23 http://www.mmarray.org
} 
Table 1

HCG CO(1-0) Galaxy Properties

\begin{tabular}{|c|c|c|c|c|c|c|c|c|c|c|c|c|c|}
\hline $\begin{array}{l}\text { HCG } \\
\text { Name }\end{array}$ & $\begin{array}{l}\text { Principal } \\
\text { Name }\end{array}$ & $\begin{array}{l}\text { R.A. } \\
\text { (J2000) }\end{array}$ & $\begin{array}{l}\text { Decl. } \\
\text { (J2000) }\end{array}$ & $\begin{array}{l}\text { Dist. }^{a} \\
\text { (Mpc) }\end{array}$ & $\begin{array}{l}\text { Morph. }^{b} \\
\text { t-type }\end{array}$ & $\begin{array}{c}F_{1.4 \mathrm{GHz}} \\
(\mathrm{mJy})\end{array}$ & {$[\mathrm{C}$ II]? } & MOHEG $^{\mathrm{c}}$ & $\begin{array}{c}\text { Optical } \\
\text { Sequence }\end{array}$ & $\begin{array}{l}\text { Spitzer } \\
\text { gap }\end{array}$ & $\begin{array}{l}\text { WISE } \\
\text { IRTZ }\end{array}$ & $\begin{array}{l}\text { Optical }^{\mathrm{d}} \\
\text { AGN }\end{array}$ & $\begin{array}{c}\mathrm{CO}^{\mathrm{e}} \\
\text { Morph. }\end{array}$ \\
\hline HCG 25b & PGC012539 & 032045.41 & -010240.9 & 85.8 & $0.9 \pm 1.3$ & $5.6^{a}$ & $\checkmark$ & $\checkmark$ & red & $\checkmark$ & $\checkmark$ & $\checkmark$ & $\mathrm{D}$ \\
\hline $\mathrm{HCG} 40 \mathrm{c}$ & PGC027508 & 093853.61 & -045136.6 & 94.1 & $2.3 \pm 2.1$ & $10.3^{b}$ & $\ldots$ & $\ldots$ & green & $\ldots$ & $\ldots$ & $\checkmark$ & $\mathrm{B}+\mathrm{R}$ \\
\hline HCG 47a & UGC05644 & 102546.26 & +134300.7 & 141 & $2.4 \pm 1.2$ & $13.1^{a}$ & $\ldots$ & $\ldots$ & blue & $\ldots$ & $\ldots$ & $\checkmark$ & $\mathrm{R}, \mathrm{S}$ \\
\hline HCG 55c & PGC035573 & 113205.69 & +704838.7 & 222 & $1.3 \pm 1.3$ & $\ldots$ & $\checkmark$ & $\ldots$ & green & $\ldots$ & $\ldots$ & $\ldots$ & $\mathrm{D}$ \\
\hline HCG 57a & NGC3753 & 113753.90 & +215853.0 & $127^{\mathrm{f}}$ & $2.1 \pm 0.6$ & $3.8^{a}$ & $\checkmark$ & $\checkmark$ & red & $\checkmark$ & $\checkmark$ & $\checkmark$ & M \\
\hline HCG 57d & NGC3754 & 113754.92 & +215907.8 & $127^{\mathrm{f}}$ & $3.4 \pm 1.1$ & $\ldots$ & $\checkmark$ & $\ldots$ & blue & $\ldots$ & $\ldots$ & $\ldots$ & $\mathrm{R}$ \\
\hline HCG 68a & NGC5353 & 135326.69 & +401658.9 & 34.6 & $-2.0 \pm 0.7$ & $40.5^{a}$ & $\ldots$ & $\checkmark$ & green & $\ldots$ & $\ldots$ & $\checkmark$ & $\mathrm{D}$ \\
\hline HCG $68 b$ & NGC5354 & 135326.70 & $\begin{array}{l}+401809.9 \\
\end{array}$ & 38.1 & $-2.1 \pm 0.7$ & $8.0^{a}$ & $\checkmark$ & $\checkmark$ & $\ldots$ & $\ldots$ & $\ldots$ & $\ldots$ & $\ldots$ \\
\hline HCG 79a & NGC6027A & 155911.14 & +204517.5 & 64.5 & $0.3 \pm 2.1$ & $9.3^{a}$ & $\checkmark$ & $\ldots$ & red & $\ldots$ & $\checkmark$ & $\checkmark$ & $\mathrm{D}$ \\
\hline HCG $82 b$ & NGC6163 & 162827.91 & +325047.0 & 148 & $-1.7 \pm 1.1$ & $\ldots$ & $\ldots$ & $\checkmark$ & red & $\checkmark$ & $\checkmark$ & $\checkmark$ & M \\
\hline HCG 91a & NGC7214 & 220907.68 & -274834.1 & 92.6 & $4.5 \pm 0.7$ & $29.2^{c}$ & $\checkmark$ & $\ldots$ & blue & $\ldots$ & $\ldots$ & $\checkmark$ & $\mathrm{S}$ \\
\hline HCG 95c & PGC071077 & 231931.09 & +093010.7 & 158 & $9.0 \pm 2.0$ & $4.10^{d}$ & $\checkmark$ & $\checkmark$ & green & $\checkmark$ & $\ldots$ & $\checkmark$ & M \\
\hline HCG 96a & NGC7674 & 232756.72 & +084644.5 & $116^{\mathrm{f}}$ & $3.8 \pm 0.6$ & $221.0^{a}$ & $\checkmark$ & $\ldots$ & blue & $\ldots$ & $\ldots$ & $\checkmark$ & $\mathrm{B}+\mathrm{R}, \mathrm{S}$ \\
\hline HCG 96c & PGC071505 & 232758.78 & +084658.1 & $116^{\mathrm{f}}$ & $5.5 \pm 4.7$ & $0.85^{d}$ & $\checkmark$ & $\ldots$ & green & $\ldots$ & $\ldots$ & $\ldots$ & D \\
\hline HCG 100a & NGC7803 & 000119.97 & +130640.5 & 69.5 & $0.1 \pm 1.0$ & $12.3^{a}$ & $\checkmark$ & $\ldots$ & blue & $\ldots$ & $\ldots$ & $\checkmark$ & D \\
\hline
\end{tabular}

Notes.

${ }^{a}$ Luminosity distance determined using the Nearby Extragalactic Database (NED).

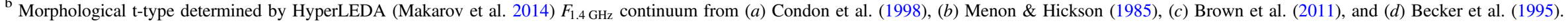

${ }^{c}$ MOHEG definition based on $\mathrm{H}_{2} / 7.7 \mu \mathrm{m} \geqslant 0.04$ (Ogle et al. 2006; Cluver et al. 2013), all except 57d are detected in $\mathrm{H}_{2}$.

${ }^{\mathrm{d}}$ Optical AGN classification from Martinez et al. (2010), counting both transition objects (TO), LINERs, and Seyferts.

${ }_{\mathrm{f}}^{\mathrm{e}}$ Morphological class of the molecular gas: $\mathrm{D}=$ disk, $\mathrm{R}=$ ring, $\mathrm{M}=$ mildly disrupted, $\mathrm{B}+\mathrm{R}=$ bar $/$ ring, and $\mathrm{S}=$ spiral, based on the classification scheme from Alatalo et al. (2013).

${ }^{f}$ Distance determined by the more massive of the group members. 
Table 2

CARMA CO $(1-0)$ Observing Parameters

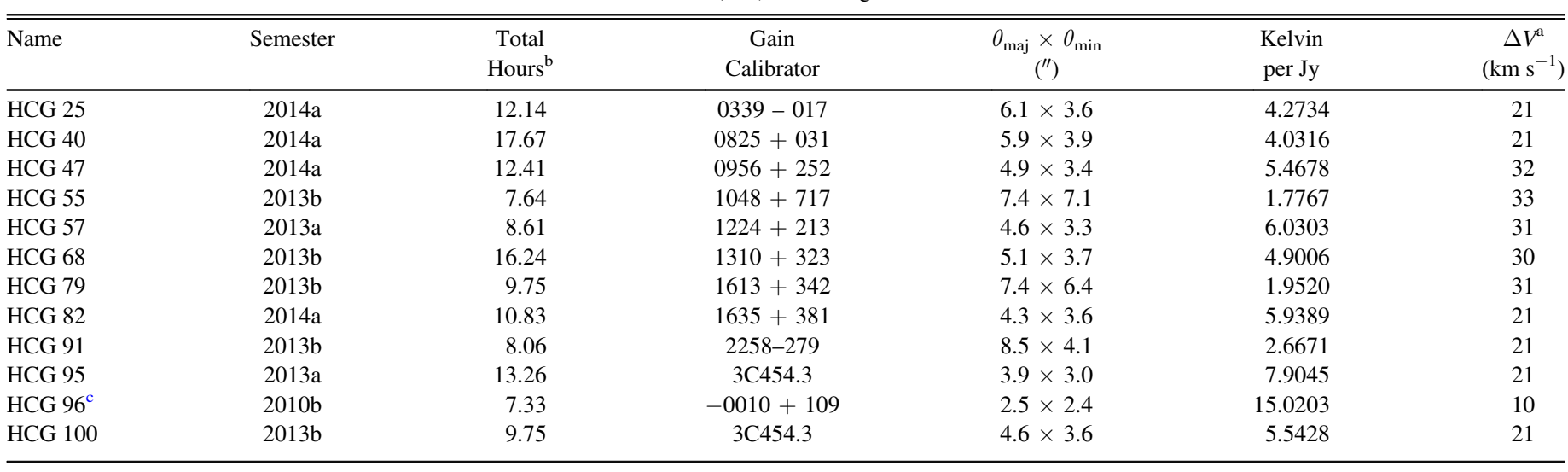

Notes.

${ }^{a}$ Channel width.

b Total time on-source time.

c Archival CARMA data.

The galaxies chosen for the new CARMA imaging were drawn from the HCGs that were detected in warm $\mathrm{H}_{2}$ using the Spitzer IRS ${ }^{24}$ (usually with boosted $L_{\mathrm{H}_{2} \text {,warm }} / L_{7.7 \mu \mathrm{mPAH}}$ ratios; Ogle et al. 2007; Cluver et al. 2013) as well as detected in CO(1-0) with the Institut de Radioastronomie Millimétrique (IRAM) $30 \mathrm{~m}$ (Lisenfeld et al. 2014). Only 10\% of HCG galaxies are MOHEGs (Cluver et al. 2013). From this subset of CO-bright, $\mathrm{H}_{2}$-strong galaxies, we chose a subset that maximized the overlap with Herschel [C II]-detected objects (P. N. Appleton et al. 2015, in preparation). These objects also tended to show early-type morphologies based on their t-types from HyperLEDA ${ }^{25}$ (Makarov et al. 2014). Eleven HCGs altogether were chosen for the CARMA sample, including eight HCGs with corresponding [C II] and [O I] Herschel observations. HCG96, although not observed through the Spitzer HCG program, had data in both the CARMA and Herschel archives, and was therefore added to our sample. All sample galaxies and their properties are listed in Table 1.

\subsection{CARMA}

The HCGs were observed with CARMA, an interferometric array of 15 radio dishes $(6 \times 10.4 \mathrm{~m}$ and $9 \times 6.1 \mathrm{~m})$ located in the Eastern Sierras in California (Bock et al. 2006). Observations were taken over the course of three semesters between 2013 March 12 and 2014 June 16. One, HCG 96, was taken from the archive. Thus, we observed a total of 12 HCGs (and 15 individual galaxies, including HCG $68 \mathrm{~b}$, detected only in $3 \mathrm{~mm}$ continuum). ${ }^{26}$ The observing strategy and data reduction were performed in a manner identical to that of the ATLAS ${ }^{3 D}$ galaxies in Alatalo et al. (2013). Table 2 presents the semester, gain calibrator, bandpass calibrator, total hours on source, and beam full-width at half-maximum (FWHM) for each of the HCGs observed with CARMA in the D-array (with baselines between 11-150 m). Galaxies with new CARMA observations

\footnotetext{
${ }^{24}$ One galaxy, HCG 57d, was not included in the original Spitzer IRS footprint.

25 http://leda.univ-lyon1.fr/

${ }^{26}$ HCG 40e was also tentatively detected, but below a signal-to-noise ratio of 3 , and only a small subset of the velocity structure was recovered.

27 The observations of HCG 96 were taken with a correlator configuration of $5 \times 250$ and $3 \times 500 \mathrm{MHz}$ per sideband, totalling $5500 \mathrm{MHz}$ of bandwidth.
}

had a correlator configuration of $8 \times 500 \mathrm{MHz}$ window in each sideband, ${ }^{27}$ with the $\mathrm{CO}(1-0)$ line utilizing the highest resolution $500 \mathrm{MHz}$ mode (with channel resolution of $\approx 15$ $\mathrm{km} \mathrm{s}^{-1}$ ). This meant there was a sufficiently large bandwidth to measure $3 \mathrm{~mm}$ continuum, which was successfully detected in $7 / 15$ sources (listed in Table 3 ). Continuum contributions were subtracted in $u v$-space using the MIRIAD task uvlin (Sault et al. 1995), as detailed in Alatalo et al. (2013).

The resulting channel maps and moment maps were also constructed in identical fashion to Alatalo et al. (2013). Figures 7-18 showcase the CARMA data for each individual $\mathrm{HCG}$, including the channel maps, integrated intensity (moment0), and mean velocity (moment1) maps, as well as integrated spectra and position-velocity diagrams (PVD). The PVD was constructed by creating a slice in the velocity cube at a certain position angle (shown as a dashed line on the moment0 figure), and integrating across a slice in space.

The integrated spectrum was constructed by using the moment0 map to create a clip-mask and integrating the flux within the moment0-defined (unmasked) aperture. This was done separately for each galaxy. The root mean square (rms) noise was then taken by calculating the standard deviation of all pixels in the cube that were outside of the moment 0 -aperture per channel and is listed in Table 3. An additional noise correction of $30 \%$ was also added in quadrature to the rms noise to account for the oversampling of the maps (see: Alatalo et al. 2015a for details). The rms noise per channel for the integrated spectrum was then calculated by multiplying the rms of the entire data cube by the square root of the total number of beams in the moment0-aperture.

To calculate an integrated line flux for each galaxy, we determined the extent of emission within the cube (shaded blue in the integrated spectrum) and summed across them. The line flux rms was then calculated by multiplying the rms per channel of the spectrum by the velocity width (listed in Table 2) and the square root of the total number of channels integrated to derive the line flux.

Table 3 lists the properties derived from the imaging data of the individually detected galaxies, including the rms noise in the channel maps, the detected continuum levels, and the total detected CO line fluxes. The spatial extent of the molecular gas 
Table 3

CARMA Derived Properties

\begin{tabular}{|c|c|c|c|c|c|c|c|c|c|c|c|}
\hline \multirow[t]{2}{*}{ Name } & \multirow{2}{*}{$\begin{array}{c}v_{\text {sys }} \\
\left(\mathrm{km} \mathrm{s}^{-1}\right)\end{array}$} & \multirow[t]{2}{*}{$z_{\mathrm{co}}$} & \multirow{2}{*}{$\begin{array}{l}\text { Vel. Range } \\
\left(\mathrm{km} \mathrm{s}^{-1}\right)\end{array}$} & \multirow{2}{*}{$\begin{array}{c}F_{100 \mathrm{GHz}}^{\mathrm{a}} \\
(\mathrm{mJy})\end{array}$} & \multirow{2}{*}{$\begin{array}{c}\mathrm{rms}^{\mathrm{b}} \\
(\mathrm{mJy} / \mathrm{bm})\end{array}$} & \multirow{2}{*}{$\begin{array}{c}\text { Flux }^{\mathrm{a}} \\
\left(\mathrm{Jy} \mathrm{km} \mathrm{s}^{-1}\right)\end{array}$} & \multirow{2}{*}{$\begin{array}{c}L_{\mathrm{CO}} \\
\left(10^{4} L_{\odot}\right)\end{array}$} & \multirow{2}{*}{$\begin{array}{l}M\left(\mathrm{H}_{2}\right)^{\mathrm{a}, \mathrm{c}} \\
\left(10^{9} M_{\odot}\right)\end{array}$} & \multicolumn{2}{|c|}{ Area } & \multirow{2}{*}{$\begin{array}{c}\Sigma\left(\mathrm{H}_{2}\right)^{\mathrm{a}, \mathrm{c}} \\
\left(M_{\odot} \mathrm{pc}^{-2}\right)\end{array}$} \\
\hline & & & & & & & & & $\left(\square^{\prime \prime}\right)$ & $\left(\mathrm{kpc}^{2}\right)$ & \\
\hline $25 b$ & 6371 & 0.02125 & $6037-6684$ & $<0.525$ & 4.83 & $21.16 \pm 2.37$ & $1.83 \pm 0.20$ & $1.60 \pm 0.18$ & 191 & 30.4 & $52.7 \pm 5.9$ \\
\hline $40 c$ & 6419 & 0.02141 & $6085-6753$ & $<0.634$ & 6.69 & $93.32 \pm 2.97$ & $9.70 \pm 0.31$ & $8.49 \pm 0.27$ & 563 & 107.7 & $78.9 \pm 2.5$ \\
\hline $47 a$ & 9637 & 0.03215 & 9429-9844 & $<0.307$ & 3.25 & $64.56 \pm 1.89$ & $14.91 \pm 0.44$ & $13.06 \pm 0.38$ & 992 & 408.4 & $32.0 \pm 0.9$ \\
\hline $55 c$ & 15760 & 0.05257 & $15394-16125$ & $<0.595$ & 3.97 & $10.09 \pm 1.33$ & $5.67 \pm 0.74$ & $4.96 \pm 0.65$ & 247 & 233.1 & $21.3 \pm 2.8$ \\
\hline $57 \mathrm{a}$ & 8723 & 0.02910 & 8214-9231 & $<0.391$ & 3.85 & $59.16 \pm 3.94$ & $11.12 \pm 0.74$ & $9.74 \pm 0.65$ & 487 & 164.6 & $59.1 \pm 3.9$ \\
\hline $57 d$ & 8944 & 0.02983 & 8753-9134 & $<0.391$ & 3.85 & $7.50 \pm 0.33$ & $1.41 \pm 0.06$ & $1.23 \pm 0.05$ & 176 & 59.3 & $20.8 \pm 0.9$ \\
\hline $68 \mathrm{a}$ & 2249 & 0.00750 & $1457-3040$ & $\begin{array}{c}6.687 \\
\pm 0.198\end{array}$ & 5.07 & $23.65 \pm 2.16$ & $0.34 \pm 0.03$ & $0.30 \pm 0.03$ & 152 & 4.2 & $71.1 \pm 6.5$ \\
\hline $68 b^{d}$ & $\cdots$ & $\cdots$ & $\cdots$ & $\begin{array}{c}1.597 \\
\pm 0.243\end{array}$ & $\cdots$ & $\ldots$ & $\cdots$ & $\ldots$ & $\ldots$ & $\ldots$ & $\ldots$ \\
\hline $79 a$ & 4149 & 0.01384 & $3841-4457$ & $\begin{array}{c}0.756 \\
\pm 0.172\end{array}$ & 5.02 & $25.42 \pm 1.91$ & $1.25 \pm 0.09$ & $1.10 \pm 0.08$ & 382 & 35.4 & $31.0 \pm 2.3$ \\
\hline $82 b$ & 10415 & 0.03474 & $10181-10652$ & $\begin{array}{c}0.483 \\
\pm 0.126\end{array}$ & 3.69 & $12.92 \pm 1.28$ & $3.28 \pm 0.32$ & $2.87 \pm 0.28$ & 99 & 44.5 & $64.6 \pm 6.4$ \\
\hline $91 \mathrm{a}$ & 6857 & 0.02287 & $6543-7171$ & $<0.991$ & 9.53 & $173.54 \pm 4.80$ & $17.44 \pm 0.48$ & $15.28 \pm 0.42$ & 2050 & 377.4 & $40.5 \pm 1.1$ \\
\hline $95 c$ & 11540 & 0.03849 & $11270-11831$ & $\begin{array}{c}0.710 \\
\pm 0.169\end{array}$ & 4.15 & $24.74 \pm 1.66$ & $7.13 \pm 0.48$ & $6.25 \pm 0.42$ & 173 & 87.3 & $71.6 \pm 4.8$ \\
\hline $96 a$ & 8638 & 0.02881 & $8482-8793$ & $\begin{array}{c}3.911 \\
\pm 0.383\end{array}$ & 7.74 & $121.75 \pm 1.59$ & $19.09 \pm 0.25$ & $16.72 \pm 0.22$ & 533 & 150.5 & $111.1 \pm 1.5$ \\
\hline $96 \mathrm{c}$ & 8809 & 0.02938 & $8592-9025$ & $<1.150$ & 7.74 & $9.61 \pm 0.13$ & $1.59 \pm 0.02$ & $1.39 \pm 0.02$ & 18 & 5.3 & $260.3 \pm 3.5$ \\
\hline $100 \mathrm{a}$ & 5220 & 0.01741 & $4888-5551$ & $\begin{array}{c}0.429 \\
\pm 0.090\end{array}$ & 4.32 & $36.99 \pm 2.34$ & $2.11 \pm 0.13$ & $1.84 \pm 0.12$ & 343 & 36.3 & $50.7 \pm 3.2$ \\
\hline
\end{tabular}

Notes.

${ }^{a}$ Does not include $20 \%$ absolute flux calibration uncertainty.

b rms noise per channel.

${ }^{\mathrm{c}}$ Does not include $30 \%$ conversion uncertainty (Bolatto et al. 2013) in $\alpha_{\mathrm{CO}}$, assuming $X_{\mathrm{CO}}=2 \times 10^{20} \mathrm{~cm}^{-2}\left(\mathrm{~K} \mathrm{~km} \mathrm{~s}^{-1}\right){ }^{-1}$.

d Detected only in $3 \mathrm{~mm}$ continuum; in the CARMA observations, see Lisenfeld et al. (2014) for its detected CO properties based on IRAM data.

was determined by summing the total number of unmasked pixels (the moment0-aperture) in the moment maps. CO luminosities (shown in Table 3) were calculated using the luminosity distance to the source (listed in Table 1). The $\mathrm{H}_{2}$ mass was determined using the $L_{\mathrm{CO}}-M\left(\mathrm{H}_{2}\right)$ relation:

$$
M\left(\mathrm{H}_{2}\right)=1.05 \times 10^{4} \frac{S_{\mathrm{CO}} \Delta v D_{\mathrm{L}}^{2}}{1+v_{\mathrm{sys}} / c} M_{\odot},
$$

which assumes $X_{\mathrm{CO}}=2 \times 10^{20} \mathrm{~cm}^{-2}\left(\mathrm{~K} \mathrm{~km} \mathrm{~s}^{-1}\right)^{-1}$ (the mean conversion factor presented in Bolatto et al. 2013). $S_{\mathrm{CO}} \Delta v$ is the $\mathrm{CO}(1-0)$ flux (in $\mathrm{Jy} \mathrm{km} \mathrm{s}^{-1}$ ), $D_{\mathrm{L}}$ is the luminosity distance (in $\mathrm{Mpc}$ ), $v_{\text {sys }}$ is the optically defined systemic velocity (in $\mathrm{km} \mathrm{s}^{-1}$ ), and $c$ is the speed of light (in $\mathrm{km} \mathrm{s}^{-1}$ ). The time variability of flux calibrators at $3 \mathrm{~mm}$ continuum adds an additional $\approx 20 \%$ uncertainty to the $\mathrm{CO}(1-0)$ flux, and the $L_{\mathrm{CO}^{-}}$ $M\left(\mathrm{H}_{2}\right)$ conversion also carries a $30 \%$ uncertainty (Bolatto et al. 2013), imposing an additional $35 \%$ absolute flux uncertainty to the measured $\mathrm{CO}$ masses. ${ }^{28}$

\subsection{Comparison of the CARMA and IRAM Data}

Thirteen of the 14 CO-imaged HCG galaxies presented in this paper were also observed by Lisenfeld et al. (2014) using the IRAM $30 \mathrm{~m}$ (only HCG 96c was not observed). Figure 1 compares the $\mathrm{CO}(1-0)$ luminosities derived from both sets of data. The measured $\mathrm{CO}(1-0)$ luminosities from CARMA and the IRAM $30 \mathrm{~m}$ are in good agreement, confirming that the

\footnotetext{
$\overline{28}$ Errors reported in Table 3 do not include the $35 \%$ uncertainty, though it is included in error bars in all figures.
}

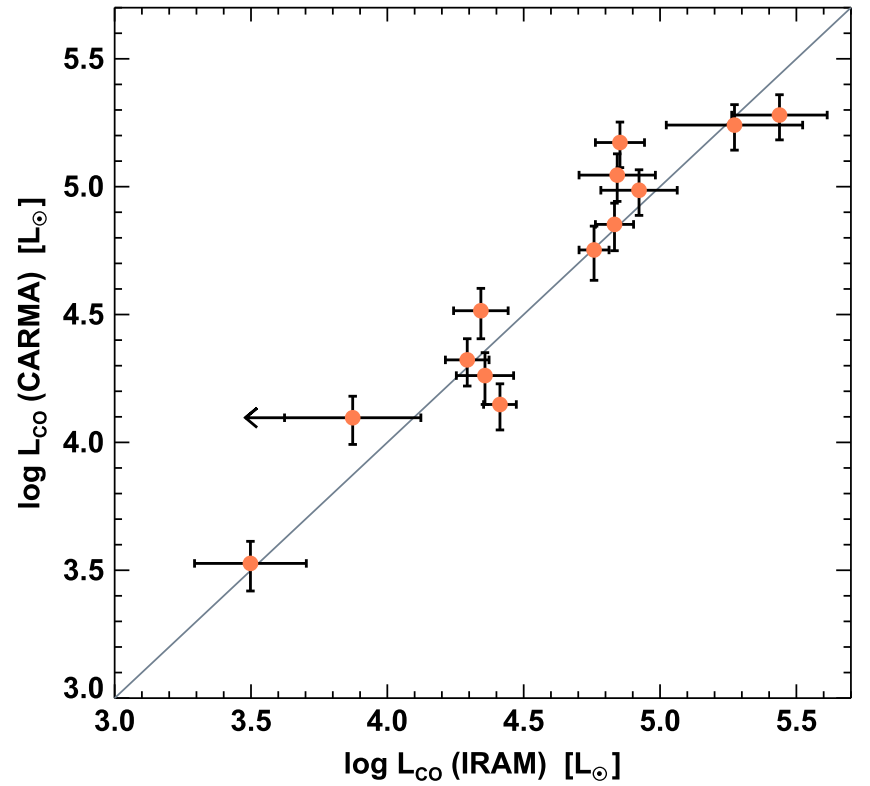

Figure 1. Comparison between the total $\mathrm{CO}$ luminosity measured by CARMA and IRAM. Errors for CARMA include both the rms and the 20\% millimeter flux calibration uncertainty. For IRAM, the range of values consists of the luminosity measured in the central pointing as a lower limit and the extrapolated total luminosity as the higher estimate. Overall, the $\mathrm{CO}$ luminosities derived from CARMA and IRAM are in good agreement.

CARMA observations do not resolve out substantial flux, and are therefore a reasonable representation of the molecular gas in these systems. HCG 57d, for which CARMA detected only half of the flux detected with the IRAM $30 \mathrm{~m}$ (possibly due to 

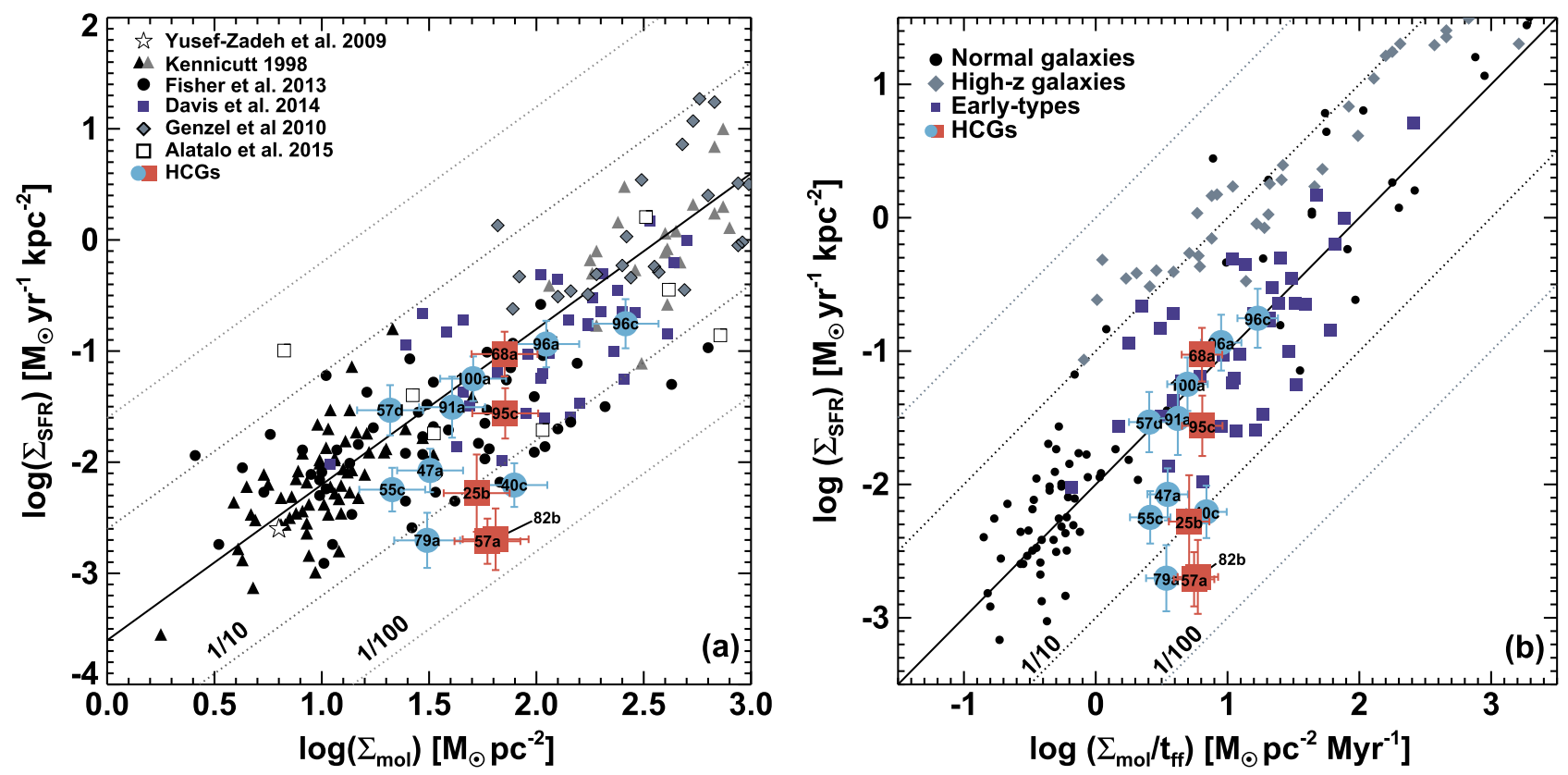

Figure 2. (Left) SFR and gas surface density in our HCG sample are shown in comparison on the Kennicutt-Schmidt relation (Kennicutt 1998). The SFRs were taken from Bitsakis et al. (2014), normalized to a Salpeter Initial Mass Function (IMF; Salpeter 1955). The HCGs are compared to the Milky Way (Yusef-Zadeh et al. 2009), normal galaxies or LIRGs (Kennicutt 1998; Fisher et al. 2013), CO-imaged early-type galaxies (Davis et al. 2014), high redshift objects (Genzel et al. 2010), and radio galaxies (Ogle et al. 2010; Alatalo et al. 2015b), all renormalized to a Salpeter IMF. The solid black line represents the Kennicutt-Schmidt relation, and dashed lines represent levels of $1 / 10$ and $1 / 100$ suppression (below) and enhancement (above). The shapes and colors of the HCG points are based on whether they meet the MOHEG criterion from Ogle et al. (2007), with red squares for HCGs with $L_{\mathrm{H}_{2} \text {,warm }} / L_{7.7 \mu \mathrm{mPAH}}>0.04$, considered confirmed MOHEGs, and blue circles representing those below this threshold. The SFR error bars include the $10 \%$ errors quoted in Bitsakis et al. (2014), and the 0.38 dex scatter in MAGPHYs-derived SFRs from Lanz et al. (2013), added in quadrature. (Right) The SF laws from Krumholz et al. (2012) are plotted for nearby and high- $z$ galaxies (black dots and gray diamonds, respectively; Krumholz et al. 2012 and references therein), and early-type galaxies (indigo squares; Davis et al. 2014), all normalized to a Salpeter IMF (Salpeter 1955). The axes of panel (b) were chosen to match the axes shown in Krumholz et al. (2012). Dotted lines represent suppressions and enhancements of 10 and 100. The HCGs found to be suppressed in panel (a) are also suppressed in panel (b).

resolving out flux, and sensitivity), is an outlier (Alatalo et al. 2014a).

\section{RESULTS}

\subsection{Molecular Gas Morphologies of the HCG Galaxies}

Following the morphological classification used by Alatalo et al. (2013) for ATLAS ${ }^{3 \mathrm{D}}$ early-type galaxies, we have morphologically classified the molecular gas in all of the $\mathrm{CO}$ imaged HCGs (listed in Table 1; detailed discussion can be found in Appendix B). We classify each galaxy as being either a disk (D), spiral (S), bar+ring $(B+R)$, ring $(R)$, mildly disrupted (M), or a combination of multiple distinct classifications. Details of the morphological classifications can be found in Alatalo et al. (2013). The morphologies seen in our HCGs tend to be a mix of regular rotation and dynamically excited structures, with a lack of strongly disrupted objects, which are quite prevalent in Ultraluminous Infrared Galaxies (ULIRGs; Sanders \& Mirabel 1996) and interacting galaxies (Wilson et al. 2008). Overall though, our CO-imaged galaxies have appearances comparable to field galaxies, including both spirals (Helfer et al. 2003) and early-types (Alatalo et al. 2013).

\subsection{The $\mathrm{K}-\mathrm{S}$ Relation in Warm, $\mathrm{H}_{2}$-bright HCG Galaxies}

Figure 2 displays the molecular gas surface density of each HCG compared with its star formation rate (SFR) surface density (calculated assuming the SF and gas are co-spatial), and using the SFR derived in Bitsakis et al. (2014). SFRs calculated by MAGPHYs (da Cunha et al. 2008) seem to have a scatter of
0.38 dex (Lanz et al. 2013), which we have included in our SF rate uncertainty. To test whether the SF histories assumed in Bitsakis et al. (2014) could significantly alter our conclusions, we input varying SF histories (including continuous and truncated models) and derived SF rates using CIGALE (Ciesla et al. 2015). The SF rates derived from cigale did not vary by more than the assumed scatter reported in Lanz et al. (2013).

$\Sigma_{\mathrm{SFR}}$ for the HCGs is compared with $\Sigma_{\mathrm{SFR}}$ of the Milky Way (Yusef-Zadeh et al. 2009), normal galaxies (Kennicutt 1998; Fisher et al. 2013), high redshift galaxies (Genzel et al. 2010), luminous infrared galaxies (LIRGs; Kennicutt 1998), radio galaxies (Ogle et al. 2010; Alatalo et al. 2015b), and COimaged early-type galaxies (Davis et al. 2014). The HCGs are color-coded based on their $L_{\mathrm{H}_{2} \text {,warm }} / L_{7.7 \mu \mathrm{mPAH}}$ ratios from Cluver et al. (2013), with MOHEGs represented by red squares, and non-MOHEGs represented by blue circles. Overall, most HCG galaxies fall within the scatter of the K-S relation (Kennicutt 1998), but HCG galaxies as a group are more likely to be found in the lower half of the scatter than the upper half (although a larger sample will be required to determine whether this is statistically significant). This result is consistent with the single-dish results from Lisenfeld et al. (2014), and there are some objects (in particular HCG 25b, 40c, 57a, 79a, and 82b) that exhibit a much more substantial scatter off of the $\mathrm{K}-\mathrm{S}$ relation. We define the degree of $\mathrm{SF}$ suppression, $\mathscr{S}$, as the ratio between the expected SFR surface density $\left(\Sigma_{\mathrm{SFR}}\right)$ from the measured molecular gas surface density $\Sigma_{\text {mol }}$ using the K-S relation (Kennicutt 1998), and the observed $\Sigma_{\mathrm{SFR}}$. Values of $\mathscr{S}$ for each HCG are listed in Table 4. 
Table 4

Star Formation Suppression Values

\begin{tabular}{|c|c|c|c|c|c|c|c|c|c|}
\hline Name & $\begin{array}{c}t_{\mathrm{ff}}^{a} \\
(\mathrm{Myr}) \\
\end{array}$ & $\mathscr{S}_{\mathrm{KS}}^{b}$ & $\mathscr{S}_{\epsilon \mathrm{ff}}^{c}$ & $\begin{array}{l}t_{\mathrm{dep}}^{d} \\
(\mathrm{Gyr})\end{array}$ & $\begin{array}{l}\sigma_{\text {norm SF }}^{e} \\
\left(\mathrm{~km} \mathrm{~s}^{-1}\right)\end{array}$ & $\begin{array}{c}\sigma_{Q=1}^{f} \\
\left(\mathrm{~km} \mathrm{~s}^{-1}\right)\end{array}$ & $\begin{array}{c}E_{\text {turb }}^{g} \\
\left(10^{55} \mathrm{erg}\right)\end{array}$ & $\begin{array}{c}L_{\text {turb }}^{h} \\
\left(10^{39} \mathrm{erg} \mathrm{s}^{-1}\right)\end{array}$ & $L_{\mathrm{H}_{2} \text {,warm }} / L_{\mathrm{turb}}^{i}$ \\
\hline $25 b$ & 10.3 & 12.2 & 9.1 & 10.0 & 91 & 51 & 7.2 & 31 & 0.34 \\
\hline $40 c$ & 11.4 & 18.2 & 10.4 & 12.7 & 104 & 62 & 57 & 303 & 0.36 \\
\hline $47 a$ & 9.1 & 3.8 & 3.9 & 3.8 & 39 & 40 & 35 & 120 & 0.50 \\
\hline $55 \mathrm{c}$ & 8.2 & 3.2 & 4.3 & 3.8 & 43 & 32 & 9.0 & 25 & 2.15 \\
\hline $57 \mathrm{a}$ & 10.6 & 38.9 & 26.8 & 30.4 & 268 & 54 & 49 & 226 & 0.82 \\
\hline $57 d$ & 8.2 & 0.6 & 0.8 & 0.7 & 8 & 32 & 2.2 & 6.0 & $\ldots$ \\
\hline $82 \mathrm{~b}$ & 10.8 & 42.2 & 27.4 & 31.9 & 274 & 56 & 16 & 76 & 0.34 \\
\hline 91a & 9.7 & 1.4 & 1.3 & 1.3 & 13 & 44 & 52 & 200 & 0.33 \\
\hline $95 \mathrm{c}$ & 11.1 & 3.6 & 2.2 & 2.6 & 22 & 59 & 37 & 193 & 0.57 \\
\hline $96 a$ & 12.4 & 1.6 & 0.7 & 1.0 & 7 & 74 & 158 & 997 & 0.14 \\
\hline $96 c$ & 15.4 & 3.4 & 0.9 & 1.5 & 9 & 113 & 31 & 297 & 0.22 \\
\hline $100 \mathrm{a}$ & 10.2 & 1.1 & 0.8 & 0.9 & 8 & 50 & 7.9 & 34 & 0.95 \\
\hline
\end{tabular}

Note. Derived SF suppression parameters for the molecular gas in HCGs including (a) the free-free timescale using Equation (2), assuming that $\sigma_{\text {gas }}=10 \mathrm{~km} \mathrm{~s}^{-1}$, $(b)$ $\mathscr{S}$ (the deviation from the K-S relation, (c) the star formation suppression from the Krumholz et al. (2012) law using $t_{\mathrm{ff}}\left(\right.$ and assuming $\sigma_{\mathrm{gas}}=10 \mathrm{~km} \mathrm{~s}{ }^{-1}$ ) and $(d)$ the depletion timescale $\left(M_{\mathrm{mol}} / \mathrm{SFR}\right)$ for each of the CO-imaged HCG galaxy. (e) The necessary molecular velocity dispersion $\left(\sigma_{\mathrm{gas}, \text { normSF }}\right)$ of the giant molecular clouds within the galaxies in order for each galaxy to have normal SF efficiency from Krumholz et al. (2012). ( $f$ ) The required global molecular gas velocity dispersion to stabilize the molecular gas against collapse (Toomre $Q>1)$. $(g)$ The corresponding total turbulent energy injection required to stabilize the molecular disk. $(h)$ The turbulent luminosity (the total turbulent energy injected per rotational period). (i) The ratio of the total warm $\mathrm{H}_{2}$ luminosity from Spitzer IRS (Cluver et al. 2013) to the required turbulent luminosity.

The molecular gas depletion (the molecular gas mass divided by the SFR) timescales associated with the HCG galaxies are also shown in Table 4 . As expected, objects with normal $\mathscr{S}$ appear to have depletion timescales similar to those found in normal, star-forming galaxies ( $\sim 1$ Gyr; Bigiel et al. 2008; Saintonge et al. 2011b), and early-type galaxies (Davis et al. 2014). The objects with $\mathscr{S}>10$ tend to have depletion timescales that are of order a Hubble time or longer, with HCG 57a and HCG 82b having depletion timescales of $>30$ Gyr. These depletion timescales suggest that these systems will nearly indefinitely contain molecular gas, if the suppressions present are continuous (rather than discrete and sporadic) processes. Given the timescale for galaxies within HCGs to transform ( 1 Gyr; Bitsakis et al. 2010, 2011; Walker et al. 2010), it is likely that the suppression is also a transient phenomenon.

In order to compare to early-type galaxies, another suppressed set of objects, we have calculated $\mathscr{S}$ for $\mathrm{CO}(1-0)$ imaged ATLAS $^{3 \mathrm{D}}$ galaxies (Young et al. 2008; Crocker et al. 2011; Alatalo et al. 2013; Davis et al. 2014). We calculated the total SFRs of these objects using the (uncorrected) WISE $22 \mu \mathrm{m}$ emission, ${ }^{29}$ assuming that the majority of the $22 \mu \mathrm{m}$ emission originates from SF in most CO-bright objects without strong AGNs (Calzetti et al. 2007; Nyland et al. 2015 ). In the CO-imaged subsample ATLAS ${ }^{3 \mathrm{D}}$ galaxies from Davis et al. (2014), we find $\mathscr{S} \approx 2.6$, consistent with the predictions of Martig et al. (2013) that the bulges in early-type galaxies can stabilize molecular disks, creating suppressions of $2-5$.

For the HCGs in this work, we find the mean $\left\langle\mathscr{S}_{\mathrm{HCG}}\right\rangle \approx 10 \pm$ 5. There also appears to be a marked bimodality seen between galaxies forming stars at normal efficiency, and a few that are extremely inefficient (with $\mathscr{S}>10$ ), including HCG $25 \mathrm{~b}, 40 \mathrm{c}$,

\footnotetext{
29 The scatter associated with the $K_{s}$-band based factor used to correct the $22 \mu \mathrm{m}$ emission for aged stars from Davis et al. (2014) is almost as large as the observed inefficiency.
}

57a, 79a, and 82b. Given the compact nature of the molecular gas and strong X-ray emission in HCG 68a (Liu 2011), it is quite possible that a non-negligible fraction of its far-infrared emission originates from the AGN, rather than from SF, similar to NGC 1266 (Alatalo et al. 2015b). The contributions of AGNs to the spectral energy distributions of individual galaxies is discussed in Appendix A.

We have also plotted the SF relation following the procedure in Krumholz et al. (2012). These authors claim that the SF rate in normal (non-LIRG) molecular configurations is dependent on the local freefall time of each individual molecular cloud, converting about $1 \%$ of the molecular mass to stars per freefall time. Krumholz et al. (2012) argue that since the majority of stars form in giant molecular clouds (GMCs), their individual properties are the dominant determinant of the SF efficiency in the molecular gas of a galaxy, and thus the local GMC freefall time is more important than the global dynamical time (as is used by Kennicutt 1998). Assuming that the GMC distribution within swathes of galaxies is fairly consistent, Krumholz et al. (2012) use this framework to universalize SF laws, across normal, star-forming galaxies and interactions. Davis et al. (2014) have also been able to show that this also applies successfully to early-type galaxies, while Utomo et al. (2015) have shown that the GMCs within example early-type galaxy, NGC 4526, matches the distributions of late-type local galaxies. We use the Krumholz et al. (2012) relation to see if our SF suppression can be reconciled by examining the GMCscale efficiencies, rather than the global scales. This framework was able to link Milky Way, local group galaxies, starburst, and high-redshift galaxy scales. The free-fall time for each HCG was calculated using Equation (4) from Krumholz et al. (2012):

$$
t_{\mathrm{ff}}=\frac{\pi^{1 / 4}}{\sqrt{8}} \frac{\sigma}{G\left(\Sigma_{\mathrm{GMC}}^{3} \Sigma_{\mathrm{gas}}\right)^{1 / 4}},
$$

where $G$ is the gravitational constant, $\Sigma_{\mathrm{GMC}}$ is the average surface density of the GMCs within the system (estimated to be 


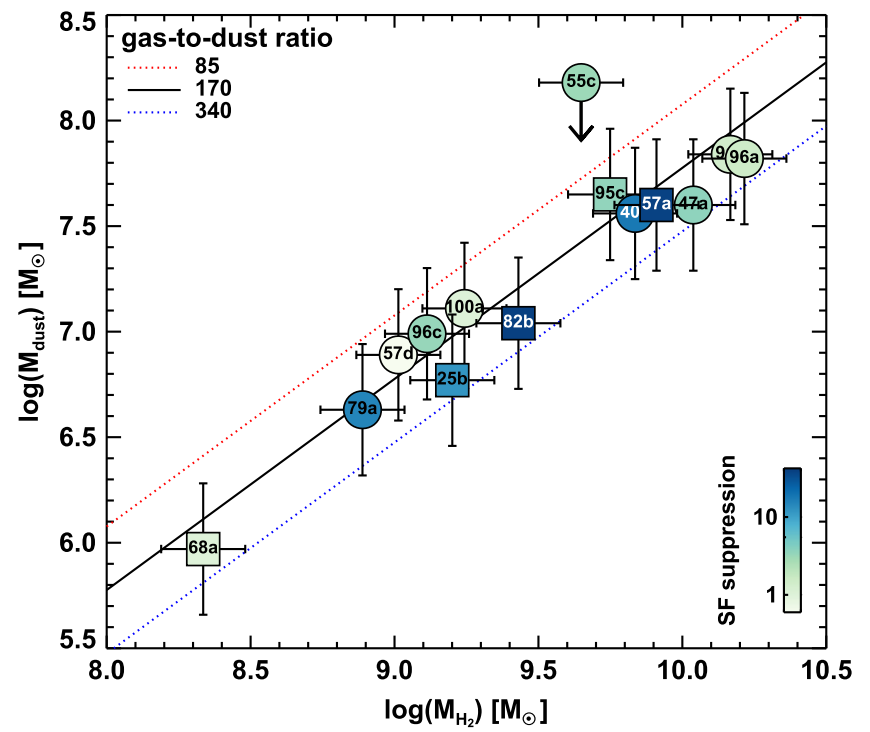

Figure 3. Molecular gas-to-dust ratios of the HCGs in this sample, using the molecular gas mass determined in this work, and the dust masses from Bitsakis et al. (2014), with an uncertainty of 0.5 dex (da Cunha et al. 2010). The dust mass of HCG 55c is an upper limit. Red and blue dotted lines represent factors of two of the average gas-to-dust ratio. The colors of the points represent the suppression of SF in the molecular gas in each galaxy. The gas-to-dust ratios of the HCGs appear consistent across a large range in molecular gas masses, as well as values of $\mathscr{S}$.

$85 M_{\odot} \mathrm{pc}^{-2}$; Bolatto et al. 2008). $\Sigma_{\text {gas }}$ for each object is presented in Table 3, and $\sigma$ is the velocity dispersion of the molecular gas within the individual GMCs, which we have assumed to be $10 \mathrm{~km} \mathrm{~s}^{-1}$ (consistent with the $8 \mathrm{~km} \mathrm{~s}^{-1}$ assumed in Krumholz et al. 2012). $\sigma_{\text {gas }}$ does not appear to vary by more than a factor of two in disk galaxies (Dib et al. 2006; Walter et al. 2008; Chung et al. 2009), though $\sigma_{\text {gas }} \approx 10 \mathrm{~km} \mathrm{~s}^{-1}$ might be an underestimate given the disrupted kinematics seen in some HCGs (Alatalo et al. 2014a). Figure 2(b) shows that some HCGs do not appear to fall on the typical efficiency (molecular mass converted into stars per free-fall time), $\epsilon \approx 0.01$ relation with the other galaxies, and those that are suppressed in the original K-S plot (Figure 2(a)) continue to appear suppressed in SF efficiency space as well. The least efficient galaxy, HCG 82b, is 27 times less efficient than the mean efficiency from Krumholz et al. (2012). A larger sample of objects with suppressions at this level is needed to be able to constrain a duty cycle and determine how long objects are observed with these large depletion times.

\subsection{Gas-to-dust Ratios in $\mathrm{H}_{2}$-bright HCG Galaxies}

Could the observed suppression in these HCGs be the result of using an incorrect $L_{\mathrm{CO}}-M\left(\mathrm{H}_{2}\right)$ conversion? Downes \& Solomon (1998) were able to show that ULIRGs did not follow the standard Milky Way relation. The molecular gas in ULIRGs is not distributed in discrete GMCs, instead being a more continuous distribution of molecular gas, and the velocity widths associated with the additional gas motions boosted the $\mathrm{CO}$ luminosity per unit gas mass. Anomalous $L_{\mathrm{CO}}-M\left(\mathrm{H}_{2}\right)$ conversion factors have also been identified in other MOHEGs, including NGC 4258 (Ogle et al. 2014) and 3C 293 (Lanz et al. 2015), and thus is an important factor to check in our sample.

In order to determine whether our observations overestimate the molecular gas mass in the suppressed HCG galaxies, we turn to the gas-to-dust ratio as a test. Figure 3 shows the gas-todust ratios of the CO-imaged HCG sample using dust masses calculated by Bitsakis et al. (2014) using full UV-to-sub-mm SED fitting in MAGPHYs (da Cunha et al. 2008). Data points are color-coded based on the galaxy's $\mathscr{S}$ value. The mean gas-todust ratio seen in our sample is $\approx 170$, within the range found for solar metallicity nearby galaxies (Sandstrom et al. 2013) and the Key Insights on Nearby Galaxies: a Far-infrared Survey with Herschel sample (Rémy-Ruyer et al. 2014). The HCG galaxies show a relationship between their dust mass and gas mass that both matches the standard value and has little-to-no dependence on $\mathscr{S}$ or classification of MOHEG. This seems to indicate that the enhanced $\mathscr{S}$ values are due in part to a physical mechanism within the molecular gas, rather than an issue with converting $\mathrm{CO}$ luminosities into molecular gas masses. Additional observations in ${ }^{12} \mathrm{CO}$ isotopologues (e.g., ${ }^{13} \mathrm{CO}$ and $\mathrm{C}^{18} \mathrm{O}$ ), as well as dense gas (e.g., $\mathrm{HCN}, \mathrm{HCO}^{+}$, and $\mathrm{CS}$ ), are necessary to confirm whether these systems require a different conversion factor, but the consistent gas-to-dust ratio appears to support a standard conversion factor.

\section{DISCUSSION}

\subsection{AGNs in the HCG Sample}

Table 1 lists the properties of each of the CO-imaged galaxies in our sample, including optical and radio signatures of AGNs. The detection of unresolved significant $3 \mathrm{~mm}$ continuum in $43 \%$ of the CARMA-imaged HCGs indicates the presence of AGNs, as the SFRs in these HCGs would not produce sufficient free-free emission at $3 \mathrm{~mm}$ continuum to be detectable by CARMA. Of the seven HCGs detected in $3 \mathrm{~mm}$ continuum, six have $1.4 \mathrm{GHz}$ radio detections as well (see Table 1 for fluxes and sources). HCG $82 \mathrm{~b}$ is the only object detected in $3 \mathrm{~mm}$ continuum but not $1.4 \mathrm{GHz}$. The optical nuclear spectra of the $3 \mathrm{~mm}$-detected HCGs all show signatures of AGNs (Table 1), either in the form of Seyfert-like spectra, low ionization nuclear emission line-region (LINER), or composite spectra (Martínez et al. 2010; Cluver et al. 2013). However, in many of these cases, slow and fast shocks might be mimicking these nuclear line ratios (Allen et al. 2008; Rich et al. 2011; Cales et al. 2015).

There is an abundance of dynamically excited features among our sample of warm $\mathrm{H}_{2}$ bright $\mathrm{HCG}$ galaxies, especially among galaxies with large values of $\mathscr{S}$ (although HCG 91a seems to also show signs of a broad blue line wing in its spectrum), which might be a sign that some of the AGNs seen are interacting with the nuclear molecular gas, possibly adding to the suppression (as is seen in NGC 1266; Alatalo et al. 2015b). Deeper analyses of these systems might reveal molecular outflows, as evidence is mounting that outflows are a common feature in objects with molecular reservoirs near an AGN (García-Burillo et al. 2014; Garcia-Burillo et al. 2015). A detailed analysis to determine whether these line wings and central broad lines are indeed due to molecular outflows would require higher resolution, higher sensitivity observations and is therefore beyond the scope of this paper. 


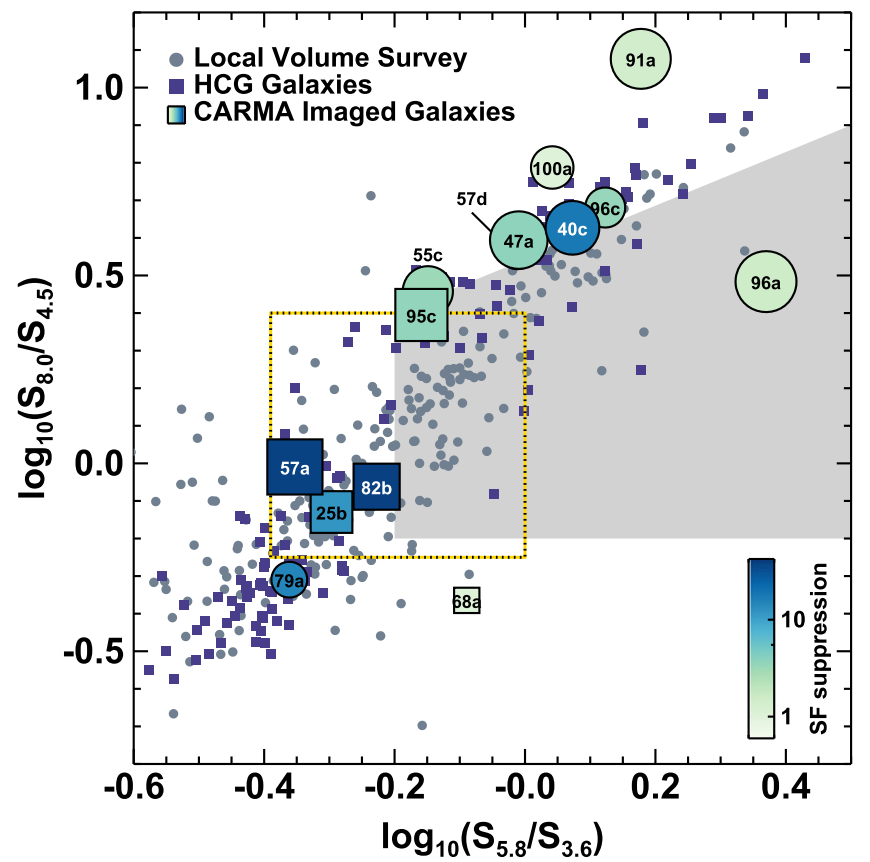

Figure 4. $S_{5.8 \mu \mathrm{m}} / S_{3.6 \mu \mathrm{m}}$ vs. $S_{8.0 \mu \mathrm{m}} / S_{4.5 \mu \mathrm{m}}$ Spitzer IRAC colors of a sample of HCGs (blue squares; Bitsakis et al. 2011; Cluver et al. 2013) compared to the Spitzer local volume legacy sample (gray dots; Dale et al. 2009), with the so-called Lacy wedge (circumscribing the AGN region in the plot) shown in light gray (Lacy et al. 2004, 2007, 2013). This plot shows the gap seen for HCGs described by Johnson et al. (2007) and Walker et al. (2010), highlighted as a yellow and black dashed line. The CO-imaged HCGs are overplotted with the color of the point representing the level of SF suppression, the size of the points representing the mass of the molecular reservoir in each HCG galaxy, and the shapes indicating whether the galaxy is a MOHEG from Cluver et al. (2013). Among the labeled HCG galaxies, squares represent MOHEGs $\left(L_{\mathrm{H}_{2}, \text { warm }} / L_{7.7 \mu \mathrm{mPAH}}>0.04\right)$ and circles non-MOHEGs. HCGs with the strongest suppression tend to be those that occupy the infrared gap in Spitzer color space.

\section{2. $\mathscr{S}$ and Its Connection to Turbulence}

Martig et al. (2013) used simulations to predict that in a set of early-type galaxies with resolved molecular gas and SF, it was possible that massive bulges served to stabilize the molecular disks in these systems against gravitational collapse, reducing the SF efficiency of the molecular gas and thus suppressing SF. For our sample, $\mathscr{S}$ does not correlate with the stellar mass of the system, the molecular gas fraction, $\left(M_{\text {mol. gas }} / M_{\text {star }}\right)$, or the visually derived morphological type of the galaxy, suggesting that an alternative driver of suppression must be at play in these systems. HCGs that fall off of the K-S relation in Figure 2(a) show similar behavior in Figure 2(b), suggesting that gravitational shears are not the main driver of the observed suppression in our sample (as was the case in early-type galaxies; Davis et al. 2014). Alternatively, the derived properties for these objects might be inaccurate. We could be underestimating $t_{\mathrm{ff}}$, for instance, because the molecular gas is not distributed in standard (Milky Way-like) molecular clouds, or because we have underestimated the molecular gas velocity dispersion (given the gas disruption that is common in HCGs). In fact, in these systems, it is possible that both diverge from the norm.

Johnson et al. (2007) noted that a fraction of galaxies within HCGs seem to undergo a rapid transition between star-forming and quiescence, with a notable lack of galaxies with intermediate infrared colors. Figure 4 plots the Spitzer Infrared
Array Camera (IRAC; Fazio et al. 2004) colors of the COimaged HCGs originally plotted in Lacy et al. (2004), overlaid with results from the Spitzer local volume legacy survey (Dale et al. 2009), as well as the HCGs from Bitsakis et al. (2011). Figure 4 shows that those of our galaxies located in the Spitzer IR gap tend to also have a high degree of SF suppression.

Cluver et al. (2013) showed that the HCG galaxies that lie within the gap (and thus are rapidly transitioning; Walker et al. 2010) also tend to be those with prominent warm $\mathrm{H}_{2}$ signatures that required mechanisms in addition to photondominated regions. Objects with $\mathscr{S} \lesssim 10$ might be able to be reconciled with normal SF of Krumholz et al. (2012) by revising our estimate of $\sigma_{\text {gas }}$ upward in the free-fall time equation, given that observations of high- $z$ galaxies show $\sigma_{\text {gas }}$ with values up to $50 \mathrm{~km} \mathrm{~s}^{-1}$ (Cresci et al. 2009). Table 4 shows what each galaxy requires to be reconciled with a normal SF efficiency. Shocks heat the gas, injecting turbulence into the system, and boosting the molecular gas velocity dispersion by factors of a few to an order of magnitude (Guillard et al. 2009). HCG 25b, 40c, 57a, 79a, and 82b have $\mathscr{S}>10$, too high to reconcile with normal SF by applying a much higher $\sigma_{\text {gas }}$.

SF requires gravitational binding energy to be greater than turbulent and radiative energies. If additional energy is introduced into the system, this balance is disrupted, leading to SF becoming inefficient (Krumholz et al. 2012). One example of turbulence-induced SF suppression in the Milky way is the galactic center cloud G0.253+0.016. This object shows evidence for a recent collision with another cloud (Longmore et al. 2012) and has a lower SFE compared to similar objects, especially in regions with high velocity dispersion (Kauffmann et al. 2013). The shock in Stephan's Quintet is detected in $\mathrm{CO}(1-0)$, but appears to have extremely weak associated SF (Konstantopoulos et al. 2014). Guillard et al. (2012a) suggest that $\mathscr{S}$ in this region could be a factor of 75 or higher, a result of the turbulence that has been generated by the shocks from the colliding galaxies. In fact, an increasing number of objects with strong turbulence have been shown to exhibit inefficient SF, including the AGN-driven molecular outflow host NGC 1266 (Alatalo et al. 2015b), and the radio galaxy 3C 326N (Guillard et al. 2015). In this sample of objects, which are known to be in collisional systems, large turbulent motions are observed associated with the suppression. The suppressed HCG galaxies in our sample seem to share some similarities with these systems, since many of them also contain gas exhibiting peculiar motions.

How much energy injection is be required in order to suppress SF? If we assume that the molecular gas in the HCG galaxies are mainly rotationally supported, we can use the Toomre criterion $(Q>1$; Toomre 1964), which describes the balance between rotation and turbulence, and gravitational binding energy, to determine the required energy budget necessary to stabilize the molecular gas against collapse, effectively inhibiting SF. To derive the necessary energy for stability $(Q=1)$, we use Equation (7) in Krumholz et al. (2012):

$$
Q=\frac{\sqrt{2(\beta+1)} \sigma \Omega}{\pi G \Sigma_{\text {mol }}}
$$

where $\sigma$ is the global gas velocity dispersion, $\Omega$ is the rotation frequency, $G$ is the gravitational constant, $\Sigma_{\mathrm{mol}}$ is the molecular gas surface density, and $\beta=0$ for the flat part of the rotation curve. We then use force balance to determine the rotation 
frequency, $\Omega$. If we assume that all disks have equivalent scale heights to the Milky Way (300 pc; Burton 1971; Malhotra 1995), the rotation frequency, $\Omega$, simplifies to:

$$
\Omega=\sqrt{\frac{3}{4 \pi} G \frac{\Sigma_{\mathrm{mol}}}{H}}
$$

where $H$ is the disk scale height. In order for the disk to be stable against collapse $(Q=1)$, assuming flat rotation, the global molecular gas velocity dispersion must be

$$
\frac{\sigma}{\mathrm{km} \mathrm{s}^{-1}} \approx 7.0\left(\frac{\Sigma_{\mathrm{mol}}}{1 M_{\odot} \mathrm{pc}^{-2}}\right)^{1 / 2}\left(\frac{H}{300 \mathrm{pc}}\right)^{1 / 2} .
$$

Assuming that the injected turbulent energy is $E_{\text {turb }}=1 / 2 \pi R_{\text {disk }}^{2} \Sigma_{\text {mol }} \sigma^{2}$, the required $E_{\text {turb }}$ must therefore be

$$
\frac{E_{\mathrm{turb}}}{\mathrm{erg}} \approx 8 \times 10^{51}\left(\frac{R_{\mathrm{disk}}}{3 \mathrm{kpc}}\right)^{2}\left(\frac{H}{300 \mathrm{pc}}\right)\left(\frac{\Sigma_{\mathrm{mol}}}{1 M_{\odot} \mathrm{pc}^{-2}}\right)^{2} .
$$

And the turbulent luminosity (energy injection rate per rotational period) is

$$
\frac{L_{\mathrm{turb}}}{\mathrm{erg} \mathrm{s}^{-1}} \approx 4.8 \times 10^{35}\left(\frac{H}{300 \mathrm{pc}}\right)^{\frac{1}{2}}\left(\frac{R_{\mathrm{disk}}}{3 \mathrm{kpc}}\right)^{2}\left(\frac{\Sigma_{\mathrm{mol}}}{1 M_{\odot} \mathrm{pc}^{-2}}\right)^{\frac{5}{2}} \text {. }
$$

Table 4 lists these values for each of the HCG galaxies in our sample. Overall, the required gas velocity dispersions to stabilize the disk of the suppressed systems are reasonable, with the total injected turbulent energy representing $\sim 1 \%$ of the total molecular kinetic energy (similar to what is seen in radio galaxies; Guillard et al. 2012b). Comparing the required turbulent injection to stabilize the disk to the extrapolated warm $\mathrm{H}_{2}$ luminosity from Cluver et al. (2013), we find that in most cases, $L_{\mathrm{H}_{2} \text {,warm }}$ is within a factor of 3 of the required turbulent injection energy (save for HCG 96a and 96d, which is a known LIRG and star-bursting system, which likely has a gravitationally unstable disk). Many of the largest $L_{\mathrm{H}_{2} \text {,warm }} / L_{\text {turb }}$ values being found in galaxies with the largest suppressions, though there is also uncertainty in the extrapolation from the Spitzer IRS footprint to the galaxy. The fact that the $\mathrm{H}_{2}$ luminosity, which is likely driven by turbulence (Appleton et al. 2006; Guillard et al. 2009, 2012a, 2012b; Cluver et al. 2013) is comparable to the turbulent luminosity seems to indicate that turbulence could indeed be the driver of the SF suppression that we are seeing in these systems, especially given the large contribution we expect from [C II] cooling (up to a factor of

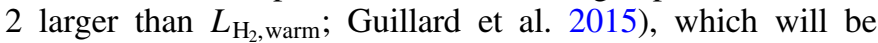
discussed in detail in an upcoming paper (P. N. Appleton et al. 2015 , in preparation).

Other mechanisms than turbulence could be responsible for the suppression of SF in those groups, like alternative gas heating or tidal disruption processes. The cosmic ray heating of the ISM may be increased in interacting galaxies (Scalo \& Elmegreen 2004), but even in systems showing extreme $\mathrm{H}_{2}$ line emission and SF suppression like Stephan's Quintet and other groups or galaxy interactions, this heating mechanism would require an extremely high cosmic ray flux (Guillard et al. 2009, 2012a; Peterson et al. 2012; Cluver et al. 2013). Alternatively, the tidal field induced by the galaxy interaction can be responsible for some expansion of the gas on the external regions of the merger, and lower the average ISM pressure (Struck 1999; Palouš 2005; Renaud et al. 2009), which could reduce the SF efficiency locally. However this effect, which depends on the geometrical configuration of the tidal field and the relative position of the galaxy with respect to this field, is difficult to quantify without a proper numerical simulation of the interaction, and is generally thought to globally increase the compressive mode of turbulence (e.g., Renaud et al. 2014).

Major mergers share many properties with HCGs, including the presence of violent interactions. In particular, the violent interactions that these galaxies are experiencing result in shocks and turbulent gas (Cresci et al. 2009). Rather than suppressing SF, most of these objects exhibit super-efficient (but short timescale) SF (Sanders et al. 1991; Sanders \& Mirabel 1996; U et al. 2012; Lanz et al. 2013). The reason HCGs, which share many of these properties, have been found with suppressed SF rather than enhanced SF is possibly the result of the different interaction timescales, larger scale environments, and gas properties. The $\mathrm{CO}(1-0)$ imaged HCG galaxies in our sample are not observed during a major merger, and they are gas-poor relative to ULIRGs.

The observed HCG galaxies have molecular gas masses and surface densities an order of magnitude smaller than what is typically seen in interacting galaxies (Downes \& Solomon 1998; Iono et al. 2009). The molecular gas in many ULIRGS is more compact compared to $\mathrm{HCG}$ galaxies as well (Bryant \& Scoville 1999; Wilson et al. 2008; Ueda et al. 2014). This likely means that although turbulence can be a disruptive force, it is also transient ( $\sim 1 \mathrm{Myr}$; Guillard et al. 2009). Without a continuous input of energy, cooling lines are able to dissipate the turbulent energy rapidly, allowing the molecular gas in ULIRGS to restart forming stars quickly, due to the higher gas densities and shorter free-fall times.

The timescale over which the violent interaction is taking place in major mergers is also short $\left(\approx 10^{8}\right.$ year; Hopkins et al. 2008; Lanz et al. 2014) compared to the total time in which gravitational forces within the compact group impact the individual galaxies ( $\approx 3$ Gyr; Plauchu-Frayn et al. 2012). In major mergers, the gravitational encounters taking place involve only the two merging galaxies (Toomre \& Toomre 1972; Privon et al. 2013). The dynamics within groups are much more complicated. HCG galaxies are interacting with the intragroup medium, and their low density gas is being ram pressure stripped (Rasmussen et al. 2008). Unlike the coalescence timescale in major mergers, galaxies in HCGs are undergoing sustained gravitational encounters with the other group members, in which collisions do not necessarily result in coalescence. This extends the timescale of turbulent injection, possibly resulting in the differences observed between major mergers and HCGs.

An in-depth analysis by Alatalo et al. (2014a) of one of the most suppressed systems, HCG 57a, suggested that an ongoing shock from a recent direct collision with HCG 57d has continuously pumped turbulence into the system. $\mathrm{H}_{2}$ and farIR cooling lines have been unable to completely dissipate the extra energy, leading to the observed SF suppression. Once the shock has traversed the system (and thus is no longer pumping energy into the molecular gas), $\mathrm{H}_{2},[\mathrm{C}$ II], and other far-IR cooling lines should efficiently cool the gas, allowing SF to return to normal efficiency. It is possible that the group environment will extend this timescale as the group members continue to interact, shocking and expelling the interstellar 


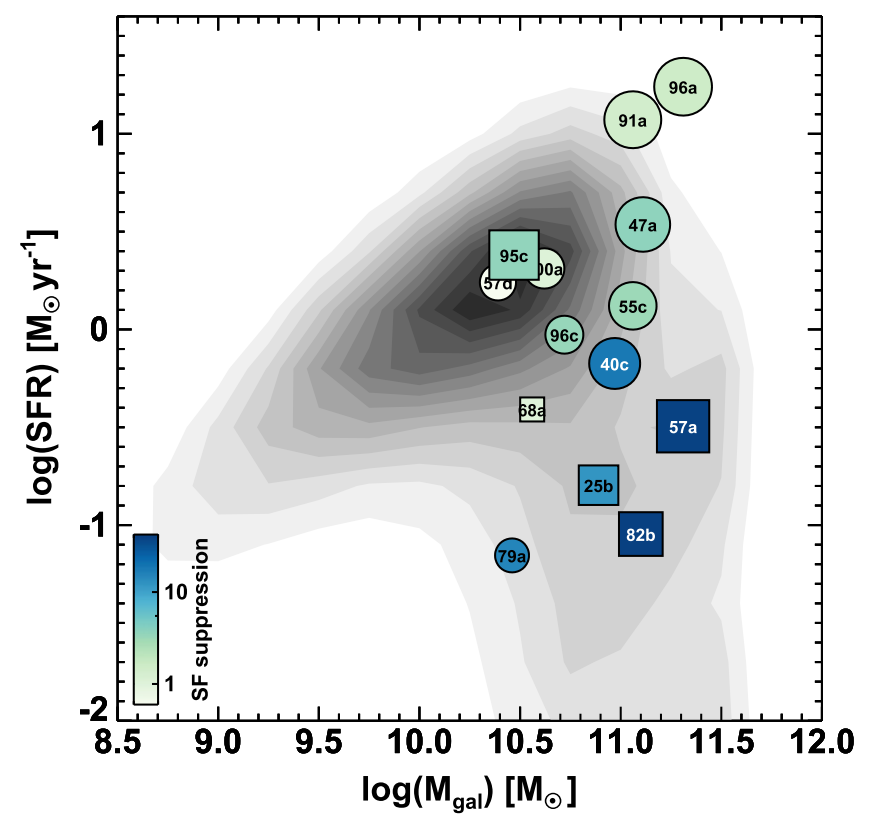

Figure 5. Galaxy mass - star formation relation of a large sample of SDSS galaxies (gray contours), with SFRs and stellar masses derived using MAGPHYS (da Cunha et al. 2008; Chang et al. 2015), showing the so-called "star formation main sequence" (Wuyts et al. 2011). Early-type galaxies are seen as the tail of high mass, low SFR. The CARMA-imaged HCGs are also overlaid, with colors coded based on their SF suppressions and symbol sizes based on their molecular gas masses. It is clear from this plot that the HCG galaxies with the most SF suppression are also the ones that are found to be farthest from the star formation main sequence.

media and ultimately transforming into early-type galaxies. This would be consistent with the compact group evolution picture suggested by Bitsakis et al. (2010) that older groups contain a higher fraction of early-type galaxies.

While it appears we have identified a sample of galaxies where turbulent energy has suppressed SF, the exact driver of this turbulence remains undetermined. Herschel data of far-IR cooling lines such as [C $\mathrm{II}]$ and [O I] will be able to advance our understanding on the interplay between the molecular gas, SF suppression, and cooling mechanisms, potentially providing us with a deeper understanding of how turbulence can impact the way in which galaxies form stars (P. N. Appleton et al. 2015, in preparation).

\subsection{SF Suppression in Warm $\mathrm{H}_{2}$-bright HCGs: A Connection to Transition?}

Figure 5 plots the stellar mass-SF main sequence for normal star-forming galaxies (Wuyts et al. 2011), compiled from the MAGPHYS-derived SFRs from SDSS DR7 (Abazajian et al. 2009; Chang et al. 2015), with our CO-imaged objects overplotted and color-coded by $\mathscr{S}$. In this phase space, the HCGs with the most SF suppression fall the farthest from the main sequence, suggesting they are transitioning. Many of the other (non-suppressed) galaxies sit on the relation, including MOHEGs. In fact, the location of objects on the main sequence seems to mirror the situation for Spitzer infrared colors (Figure 4), strongly suggesting that the most suppressed objects are also the ones that are currently transitioning between the star-forming spiral and quiescent early-type populations.
Figure 6 plots the CARMA-imaged HCG galaxies on the optical and IR color-magnitude diagrams presented in Alatalo et al. (2014b). The HCGs we have studied span a large range of optical and IR colors, although they are generally more massive than the underlying Galaxy Zoo distributions (Lintott et al. 2008; Schawinski et al. 2014). One would expect that the total molecular mass or molecular gas fraction might be the determining factor of the position of the HCGs on the color-magnitude diagrams, but that is not the case. HCG 40c and 57a both contain large reservoirs of molecular gas, while appearing in the optical green valley or red sequence (compared to HCG91a and 96a with equivalent molecular mass, which are quite blue). In fact, Figure 6 shows that the mass of the molecular reservoir does not determine the color of the galaxy. Spearman rank tests were used to search for correlations between $\mathscr{S}$ and other galaxy properties. The only galaxy property significantly correlated with $\mathscr{S}$ was color. Including HCG 68a, a Spearman correlation of -0.71 with a $p$-value $=0.0044$ was found when investigating $\mathscr{S}$ and $u-r$ color. If HCG 68a is removed, we find a $\mathscr{S}-(u-r)$ Spearman correlation of -0.71 (equal to the test with HCG 68a included) with a $p$-value $=0.0011$. Without HCG 68a, $\mathscr{S}-([4.6]-$ [12] $\mu \mathrm{m})$ also shows a Spearman correlation of 0.80 with a $p$-value $=0.0067$. With or without HCG 68a, the optical and IR colors were the only galaxy properties shown to significantly correlate with $\mathscr{S}$. The extreme IR colors of HCG 68 a might be due to a buried AGN.

These results seem to suggest that a galaxy does not need to shed its ISM to then quench SF (the standard galaxy transition picture; Hopkins et al. 2006) to morphologically transform. Instead, changing the state of the molecular gas can act to quench SF before the ISM has been completely shed. It is thus possible that in many of our CO-imaged galaxies, the molecular gas has been rendered infertile due to shocks pumping turbulence into the system. The quenching of SF, and the beginning of the transition across the green valley, occurs before the galaxy loses the majority of its molecular ISM. This is consistent with the findings of Leon et al. (1998) and Martinez-Badenes et al. (2012) that HCG galaxies contain comparable molecular gas reservoirs to isolated galaxies (Lisenfeld et al. 2011; Saintonge et al. 2011a). Alatalo et al. (2014b) posited that the IRTZ was a manifestation of evolution, representing the stage in which a galaxy is actively shedding its ISM, and that this phase follows SF quenching (traced by the $u-r$ colors). In this scenario, first the galaxies move toward the elbow in Figure 6(b), where SF is suppressed, and then they move into the IRTZ, where they quench SF and move to the optical red sequence. The H I findings of Serra et al. (2012) of that $40 \%$ of field early-type galaxies still contain nonnegligible reservoirs of neutral gas. New work on poststarburst galaxies found that many poststarburst galaxies still contain non-negligible molecular reservoirs (French et al. 2015; Rowlands et al. 2015), confirming the suggestion that SF quenching can take place before galaxies expel their molecular interstellar media. It is currently unclear how often galaxies transition in this fashion, but further observations of IRTZ and poststarburst galaxies, combined with our new observations of at least some HCG galaxies (certainly those that are warm $\mathrm{H}_{2}$-bright and $\mathrm{CO}$-bright), have shown that this "quenching first" path contributes to the population of transitioning galaxies. 


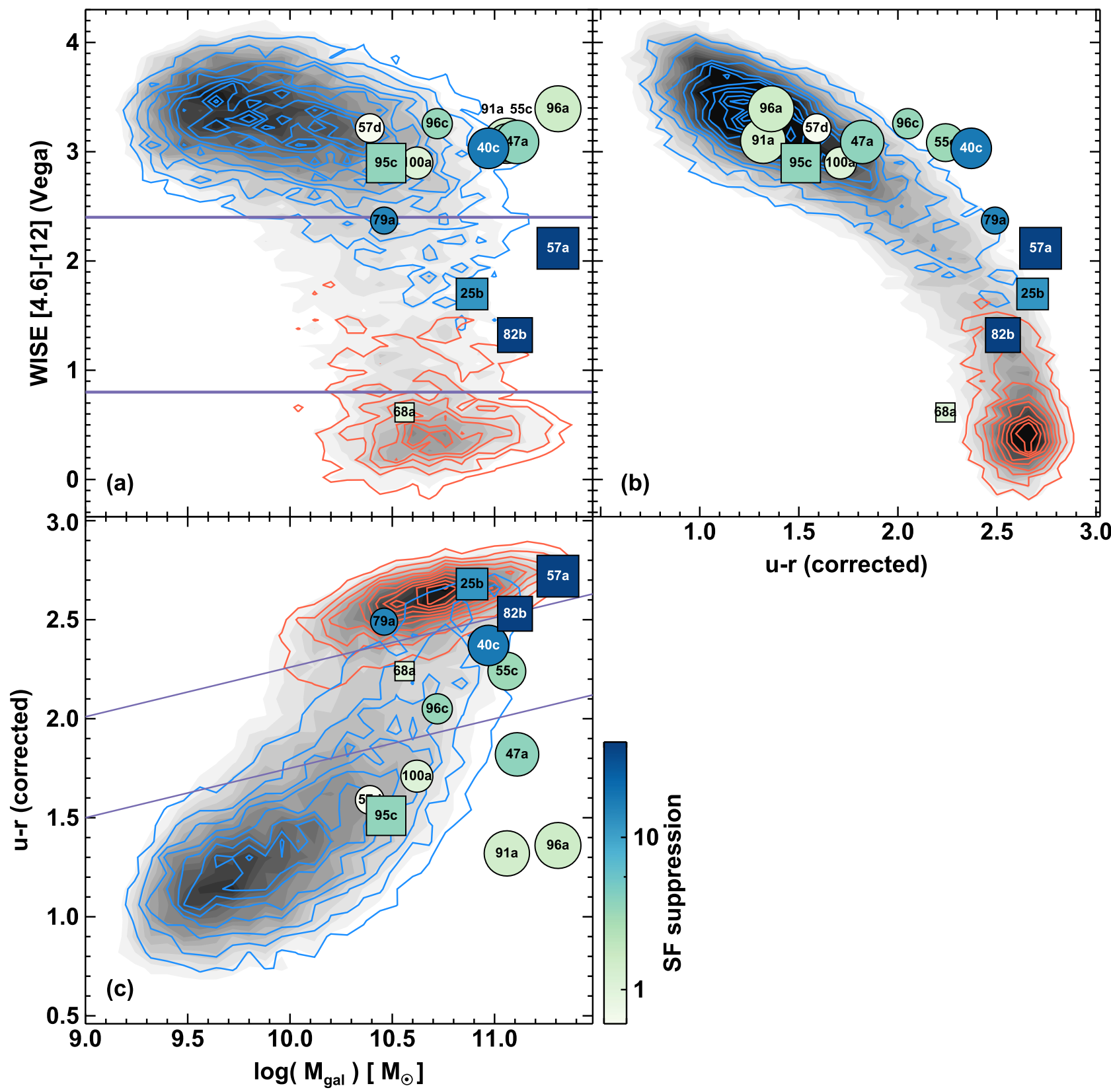

Figure 6. Early-type (red contours) and late-type (blue contours) galaxies from the Galaxy Zoo (Lintott et al. 2008; Alatalo et al. 2014b; Schawinski et al. 2014) are compared to the HCGs. The color of the points represent the level of SF suppression, the size of the points correspond to the size of the molecular reservoir, and the shapes indicate whether the galaxy is a MOHEG from Cluver et al. (2013). Squares represent MOHEGs and circles non-MOHEGs. (Top left) The stellar mass vs. WISE [4.6]-[12] $\mu \mathrm{m}$ colors, overlaid with indigo lines defining the IRTZ. (Top right) The $u-r$ vs. [4.6]-[12] $\mu \mathrm{m}$ sequence identified by Alatalo et al. (2014b), with HCGs overplotted. (Bottom) The stellar mass vs. $u-r$ colors of the Galaxy Zoo galaxies from Schawinski et al. (2014), overlaid with the segment defining the optical green valley. The HCGs that are overplotted had both optical and IR colors derived from Bitsakis et al. (2014). The most suppressed galaxies are the most likely to be in the WISE IRTZ and on or near the optical red sequence.

The presence of suppressed objects within the IRTZ points to a possible new method for identifying galaxies most likely to be in a phase of inefficient SF, before the exhaustion of their molecular gas. By directly imaging the $\mathrm{CO}$ in molecular gasrich galaxies that also appear in the IRTZ, we can pinpoint a population of galaxies likely to exhibit SF suppression. This result suggests that future studies might identify larger samples of suppressed galaxies by selecting galaxies based on their optical and IR colors. A larger sample of suppressed systems thus allows us to study how the injection of turbulence not only impacts the energy balance that dictates our SF laws (Krumholz et al. 2012), but also allows us to study how the neutral ISM is exhausted as a galaxy transforms from a spiral into an earlytype galaxy.

\section{SUMMARY}

1. We have used CARMA to map $\mathrm{CO}(1-0)$ of galaxies within 12 HCGs, many with elevated warm $\mathrm{H}_{2}$ emission, detecting molecular gas in 14 galaxies, and unresolved $3 \mathrm{~mm}$ radio continuum in 7 (consistent with the presence of AGNs). Figures 7-18 show the molecular gas data for each galaxy, including the moment0 map, moment1 map, channel maps, PVDs, and integrated spectra. A 

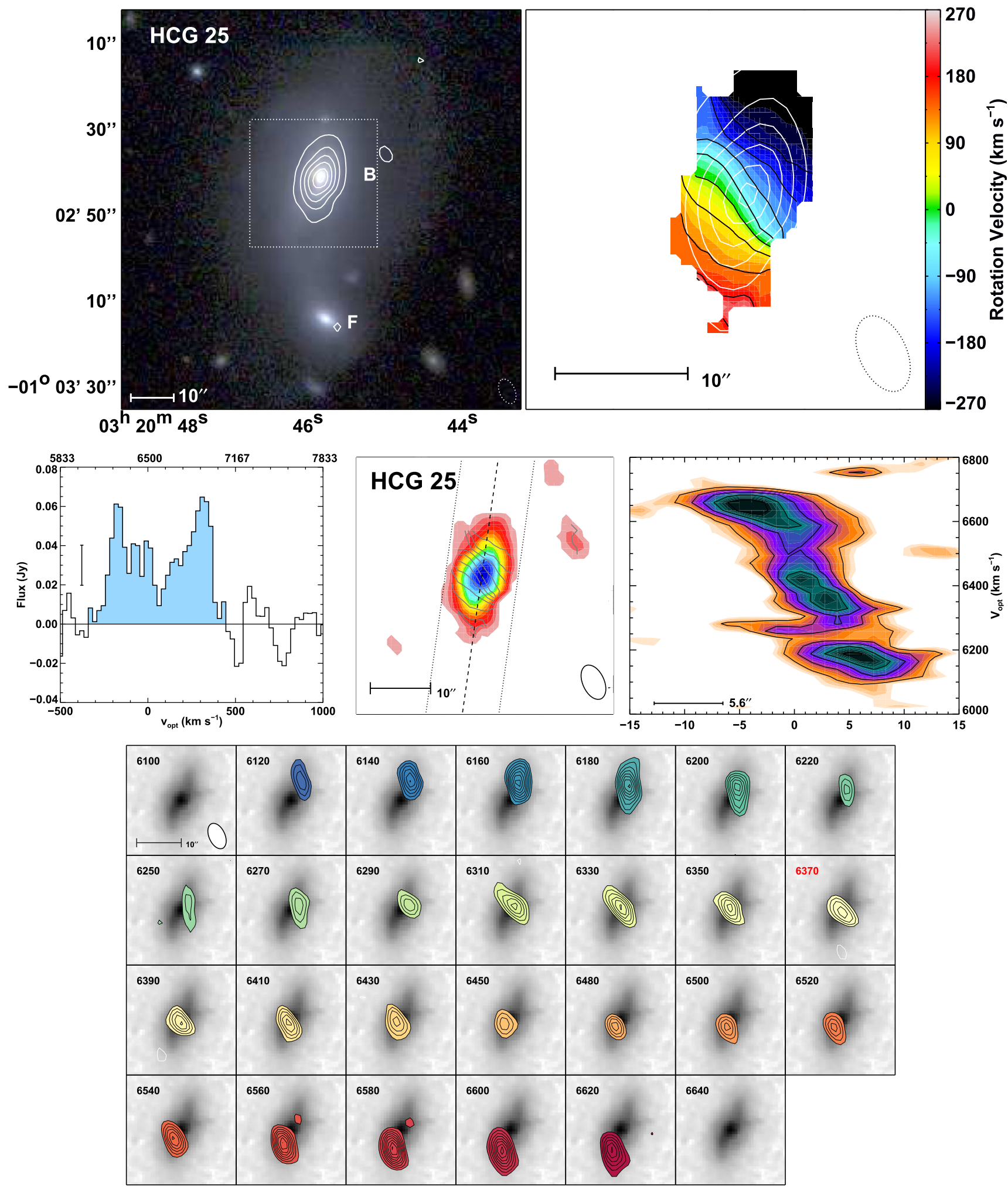

Figure 7. HCG 25. Channel map contours are in $1 \sigma$ steps. Elements of this figure are described in Appendix C.

comparison to the single-dish data from Lisenfeld et al. (2014) shows that our observations have not resolved out large fractions of the flux.

2. The molecular gas morphologies of our HCG galaxies (using the metric set for the ATLAS ${ }^{3 \mathrm{D}}$ galaxies in Alatalo et al. 2013) indicate that HCGs are consistent with the distribution of gas morphologies found in early-type and spiral galaxies rather than ULIRGs (Wilson et al. 2008; Ueda et al. 2014).

3. We have shown that a large proportion of our CO-imaged HCG galaxies exhibit SF suppression $(\mathscr{S})$ when plotted relative to both the K-S relation (Kennicutt 1998) as well as the universal SF law from Krumholz et al. (2012). The mean SF suppression for this sample is $\langle\mathscr{S}\rangle \approx 10 \pm 5$, 
and exhibits a bimodality. The most extreme objects (HCG 25b, 40c, 57a, 79a and 82b) exhibit $\mathscr{S} \gtrsim 10$, and have molecular gas depletion timescales $t_{\mathrm{dep}} \geqslant 10 \mathrm{Gyr}$.

4. The mean gas-to-dust ratio for the CO-imaged HCGs is around 170 , within the range found in normal galaxies (Sandstrom et al. 2013; Rémy-Ruyer et al. 2014). We do not believe that the observed $\mathscr{S}$ is due to an incorrect $L_{\mathrm{CO}}-M\left(\mathrm{H}_{2}\right)$ conversion factor (which would appear as a highly discrepant gas-to-dust ratio in suppressed galaxies).

5. A non-negligible fraction of our CO-imaged HCG galaxies contained substantial warm $\mathrm{H}_{2}$ emission (Cluver et al. 2013), consistent with there being shocks injecting substantial turbulence into these systems, and the turbulent energy required to stabilize the molecular gas against collapse appears to agree within an order of magnitude with the warm $\mathrm{H}_{2}$ luminosity. As has been seen in the Milky Way (Kauffmann et al. 2013), NGC 1266 (Alatalo et al. 2015b), and 3C 326N (Guillard et al. 2015), the additional turbulence could upset the energy balance that dictates the rate of SF (Krumholz et al. 2012), thereby suppressing SF.

6. HCGs with the most SF suppression are usually located within the transition regions of optical and IR color space, independent of the mass of the molecular reservoir. This ties in well with work that indicates that galaxies are able to transition in colors and quench SF before they have shed their ISMs (Alatalo et al. 2014b; French et al. 2015; Rowlands et al. 2015), showing how galaxies might render their molecular reservoirs infertile before expelling them. This could play an important role in understanding the blue to red galaxy transition.

7. The $u-r$ and WISE IRTZ colors, combined with a CO detection, are also able to select the objects most likely to exhibit SF suppression, providing an ideal sample selection criterion with which to study this phenomenon.

We thank Laure Ciesla for lending her advice and CIGALE expertise in our investigation of the SF uncertainties, as well as Philip Chang for prescient advice on the theoretical grounding of the energy budget. We also thank the anonymous referee for a useful and insightful report. Partial support was provided to K.A., T.B., and P.A. by NASA observations through a contract issued by the Jet Propulsion Laboratory, California Institute of Technology under a contract with NASA. Additional support for K.A. is provided by NASA through Hubble Fellowship grant \#HST-HF2-51352.001 awarded by the Space Telescope Science Institute, which is operated by the Association of Universities for Research in Astronomy, Inc., for NASA, under contract NAS5-26555. U.L. acknowledges support by the research projects. AYA2011-24728 from the Spanish Ministerio de Ciencia y Educación and the Junta de Andalucía (Spain) grants FQM108. T.B. and V.C. would like to acknowledge partial support from the EU FP7 Grant PIRSES-GA-2012-316788. T.B. acknowledges support from DGAPA-UNAM postdoctoral fellowships. L.V.M. work has been supported by grant AYA2011-30491-C02-01 co-financed by MICINN and FEDER funds, and the Junta de Andalucia (Spain) grants P08-FQM-4205 and TIC-114.

Support for CARMA construction was derived from the Gordon and Betty Moore Foundation, the Kenneth T. and Eileen L. Norris Foundation, the James S. McDonnell Foundation, the Associates of the California Institute of
Technology, the University of Chicago, the states of California, Illinois, and Maryland, and the National Science Foundation. This publication makes use of data products from the Widefield Infrared Survey Explorer, which is a joint project of the University of California, Los Angeles, and the Jet Propulsion Laboratory/California Institute of Technology, funded by the National Aeronautics and Space Administration. The work is also based, in part, on observations made with Herschel, a European Space Agency Cornerstone Mission with significant participation by NASA. This research has made use of the NASA/IPAC Extragalactic Database (NED) which is operated by the Jet Propulsion Laboratory, California Institute of Technology, under contract with the National Aeronautics and Space Administration. We acknowledge the usage of the HyperLeda database (http://leda.univ-lyon1.fr).

Facilities: CARMA, Herschel, WISE.

\section{APPENDIX A \\ DETERMINING THE AGN CONTRIBUTIONS WITHIN CO(1-0) IMAGED HCG GALAXIES}

In Table 1, we presented the AGN classifications of the galaxies in our sample, based both in optical emission line diagnostics as well as radio continuum. Such sources can significantly contribute to the infrared emission of the galaxies, leading to an overestimation of their SFRs, if they are not accounted for. To disentangle the fraction of AGN contribution in the total infrared luminosity we have fitted the galaxy observed infrared (8-500 $\mu \mathrm{m}$; purely dust emission) spectral energy distributions with DECOMPIR (Mullaney et al. 2011). This code simply fits the observed fluxes with sets of hostgalaxy + AGN component templates, and estimates the contribution of the AGN to the total infrared luminosity. The AGN templates are described by broken power-laws at around $40 \mu \mathrm{m}$ that fall steeply above that. From this analysis we find that only three of our sources have significant AGN contribution at the infrared bands, HCG 68a with $9 \%$, and HCG 91a with 35\%.

\section{APPENDIX B \\ COMMENTS ON INDIVIDUAL GALAXIES}

$H C G 25 b$ : Figure 7 shows that HCG 25b is an edge-on galaxy. Deep optical imaging by Eigenthaler et al. (2015) also showed that HCG 25b is interacting strongly with HCG 25f. A tidal tail connects the two, and the polyaromatic hydrocarbon (PAH) emission shows a small tidal feature. HCG 25b is also a MOHEG (Cluver et al. 2013) and is a transitioning galaxy in the IR (i.e., it lies in the optical red sequence as well as the Spitzer IR gap and the WISE IRTZ). HCG 25b also likely contains an AGN, classified through optical emission line diagnostics (Martinez et al. 2010), and the presence of $1.4 \mathrm{GHz}$ nuclear emission. ${ }^{30}$ The molecular gas is morphologically classed as a disk.

$H C G 40 \mathrm{c}$ : Figure 8 shows that $\mathrm{HCG} 40 \mathrm{c}$ is an edge-on galaxy strongly detected with CARMA. While HCG 40c lies in the red part of the WISE IRTZ, it is found in the optical green valley, though near the red sequence. It also has a radio core and optically identified AGN (Martinez et al. 2010). Two Micron All-sky Survey (2MASS) imaging also shows that

\footnotetext{
${ }^{30}$ Nuclear $1.4 \mathrm{GHz}$ emission can also be due to SF (Condon 1992). Thus, detecting $1.4 \mathrm{GHz}$ emission in these objects does not confirm the presence of an AGN without morphological confirmation (Best et al. 2005) and thus are just suggestive of their presence.
} 

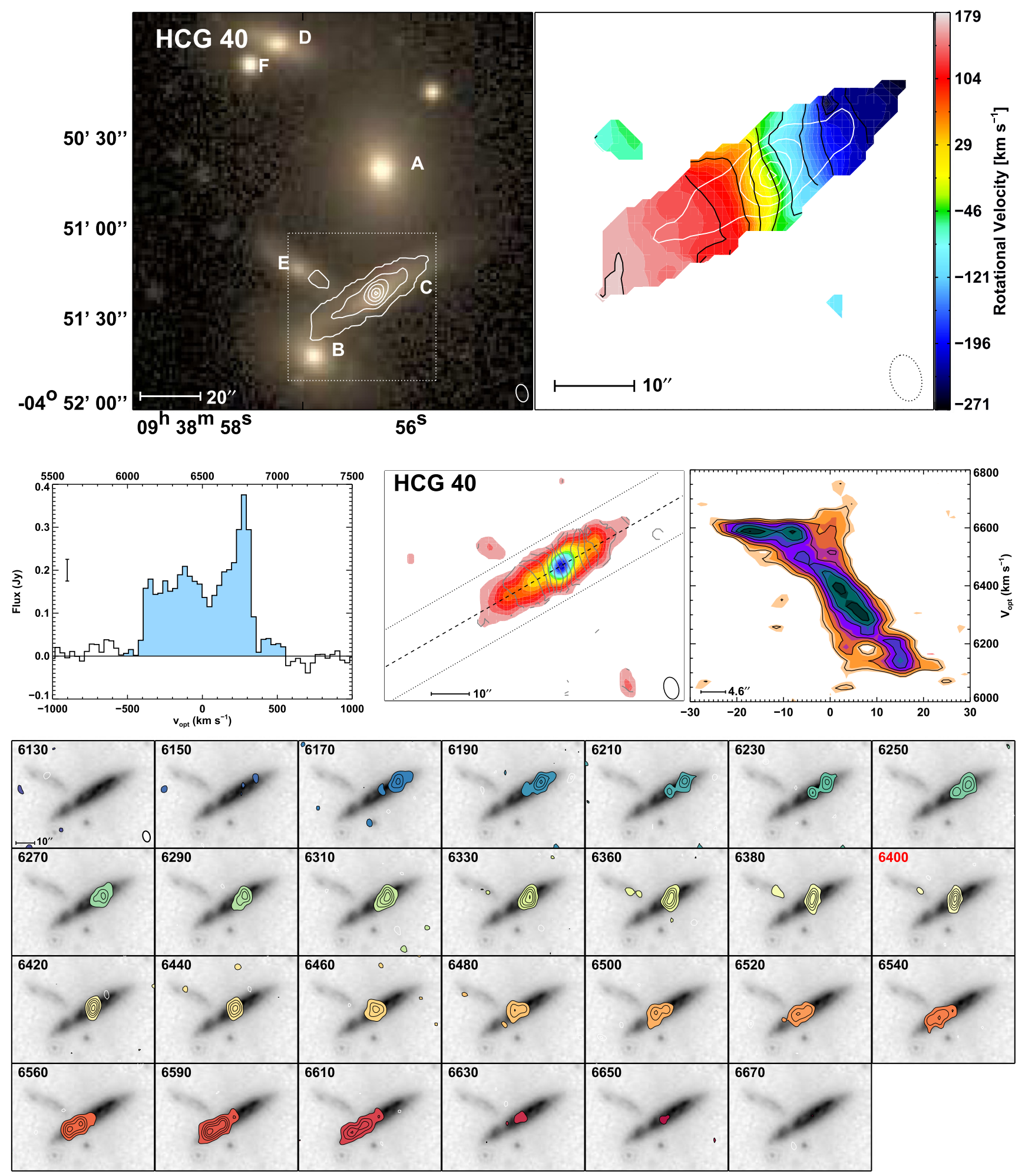

Figure 8. HCG 40. Channel map contours are in $3 \sigma$ steps.

HCG 40c is tidally interacting with HCG 40e (Skrutskie et al. 2006). The PVD of HCG 40c (Figure 8) also shows a significant bar (seen as the large velocity structure with very little position shift), similar to what was seen by Alatalo et al. (2013) in several ATLAS ${ }^{3 \mathrm{D}}$ early-type galaxies. This is likely what is responsible for the slight appearance of broad wings in the $\mathrm{CO}$ spectrum. Given the frequency with which bars arise during gravitational encounters (Athanassoula 1996;
Athanassoula \& Bureau 1999), it is unsurprising that HCG 40c is morphologically classed as a bar+ring.

HCG 47a: Figure 9 shows that HCG 47a is an oblong spiral (in both PAH and optical images from SDSS; York et al. 2000) that is tidally interacting with HCG 47b. HCG 47a is also on the cusp of being considered a MOHEG $\left(L_{\mathrm{H}_{2} \text {,warm }} / L_{7.7 \mu \mathrm{mPAH}}\right.$ $=0.035$; Cluver et al. 2013). The center, while void of molecular gas, contains a spectrally classified AGN (Stern \& 

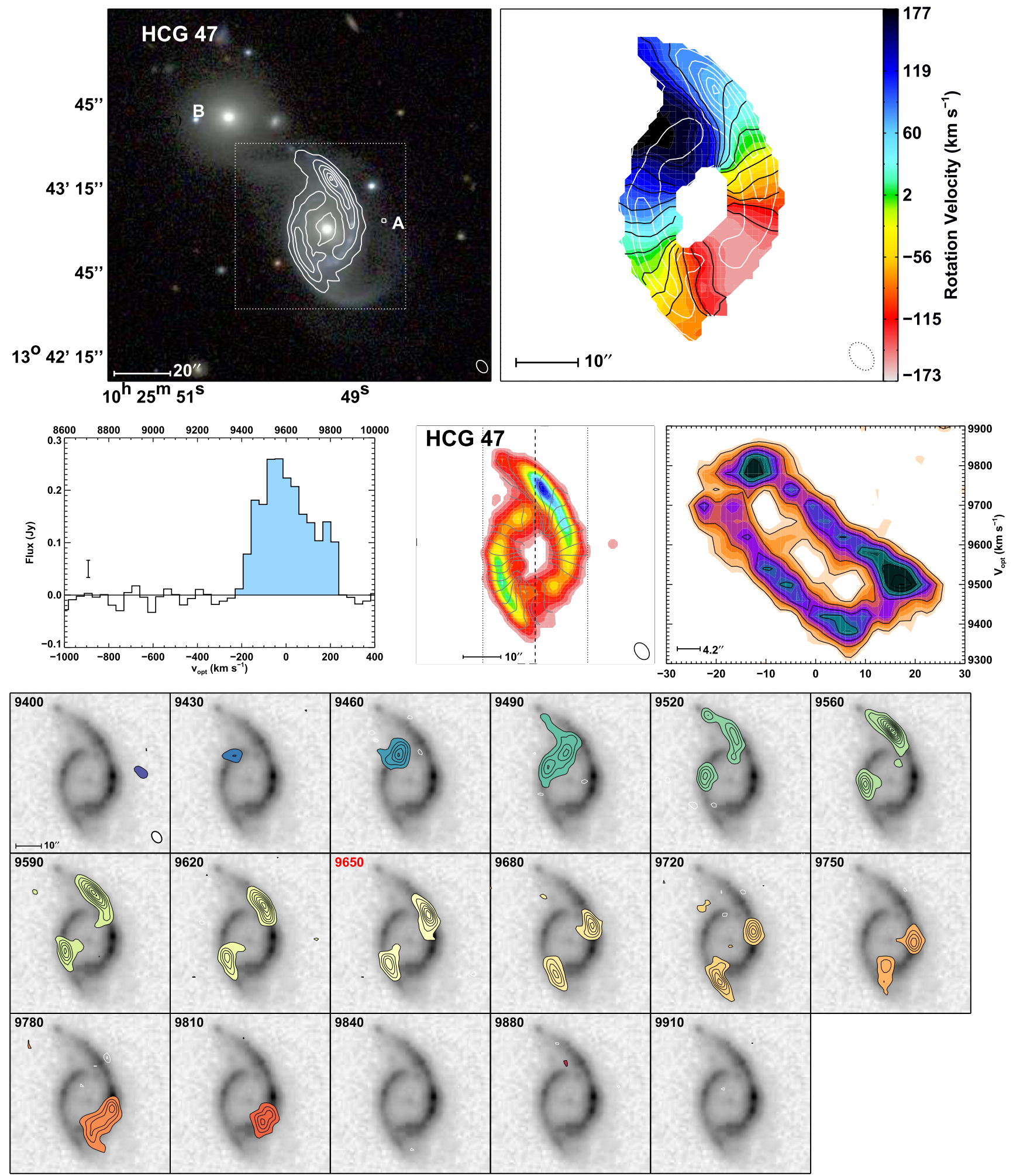

Figure 9. HCG 47. Channel map contours are in $3 \sigma$ steps.

Laor 2012) as well as $1.4 \mathrm{GHz}$ emission. The $\mathrm{CO}(1-0)$ emission traces the oblong spiral, and so the molecular gas is morphologically classified as both a ring and a spiral.

$H C G 55 c$ (Figure 10) is part of the chain of galaxies VV 172/Arp 239. ${ }^{31}$ HCG 55 is the most distant HCG that we

\footnotetext{
$\overline{31}$ It contains a galaxy with a highly discrepant redshift (HCG 55e, $v=36,880 \mathrm{~km} \mathrm{~s}^{-1}$; Sargent 1968).
}

have imaged with CARMA. The emission in this galaxy is resolved and spans approximately 3 beam widths. HCG 55c was not detected in $1.4 \mathrm{GHz}$ or $3 \mathrm{~mm}$ continuum, and does not have a published optical spectrum (to determine the presence of an AGN). However, HCG 55c is at the edge of the Spitzer gap, and is in both the optical green valley and the WISE IRTZ. The molecular gas in HCG 55c is morphologically classified as a disk. 


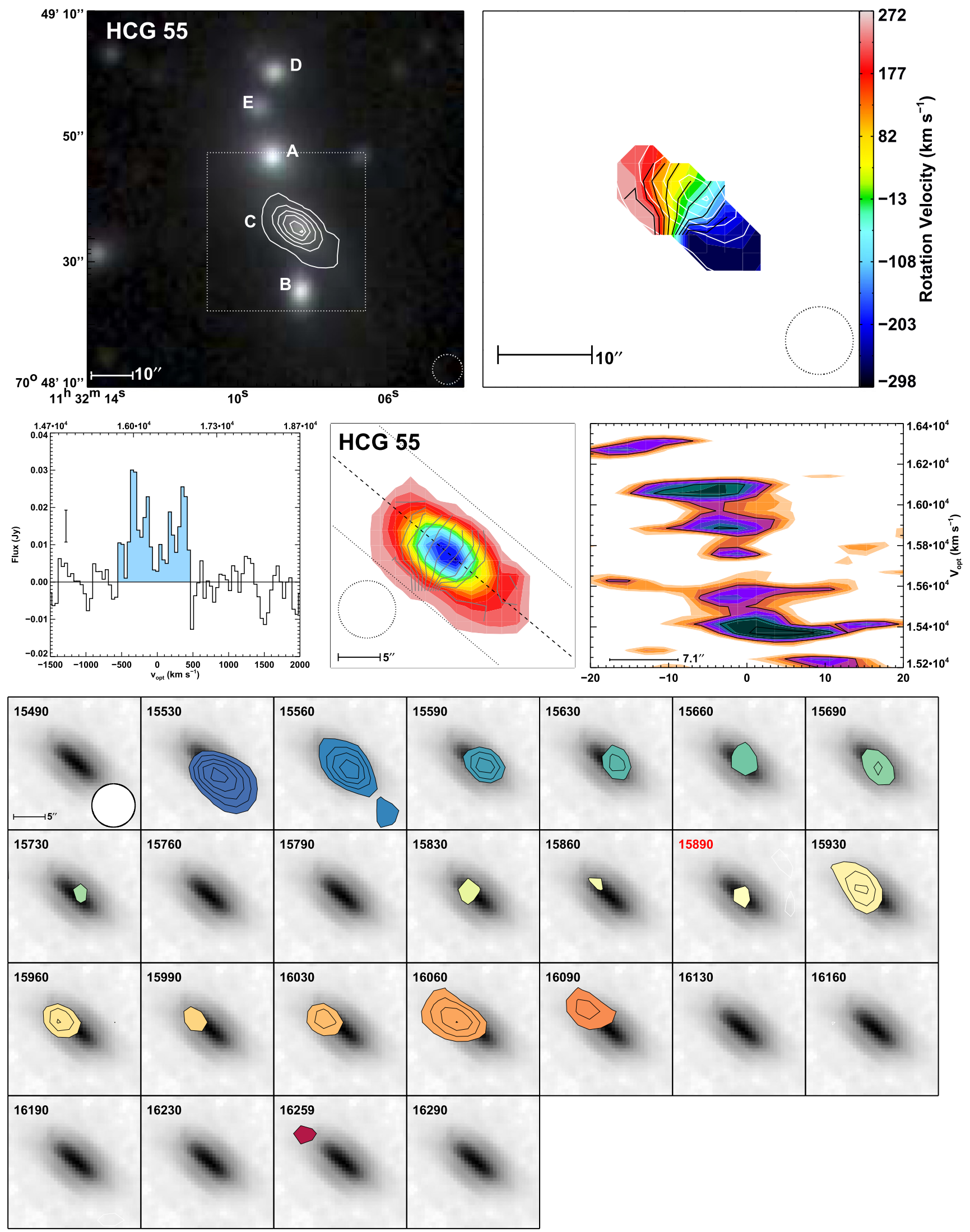

Figure 10. HCG 55. Channel map contours are in $1 \sigma$ steps. 

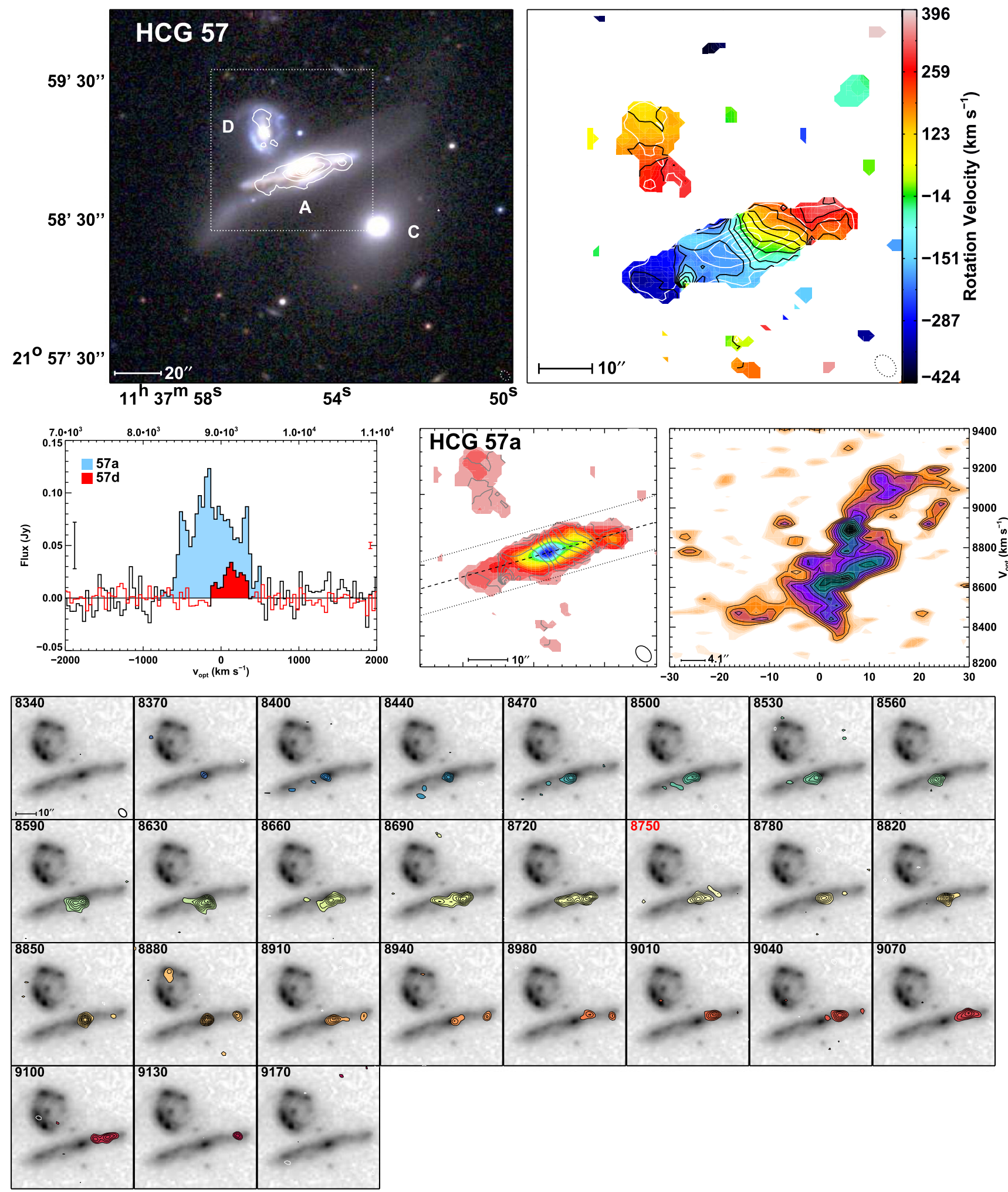

Figure 11. HCG 57. Channel map contours start at $\pm 2.5 \sigma$ and are in $1 \sigma$ steps. The PVD of $57 \mathrm{~d}$ can be found in Figure 13 .

HCG $57 a$ is found to have some of the most complex molecular gas dynamics in the sample, including three distinct kinematic components (Figure 11), and is interacting with HCG 57d. HCG 57a is also a MOHEG (Cluver et al. 2013), and an in-depth discussion of both HCG 57a and HCG 57d can be found in Alatalo et al. (2014a). The molecular gas in HCG 57a is morphologically classified as mildly disrupted.

HCG 57d (Figure 11) was not within the Spitzer IRS footprint, and thus we do not have warm $\mathrm{H}_{2}$ information on this source. An in-depth discussion of both HCG 57a and HCG 57d 

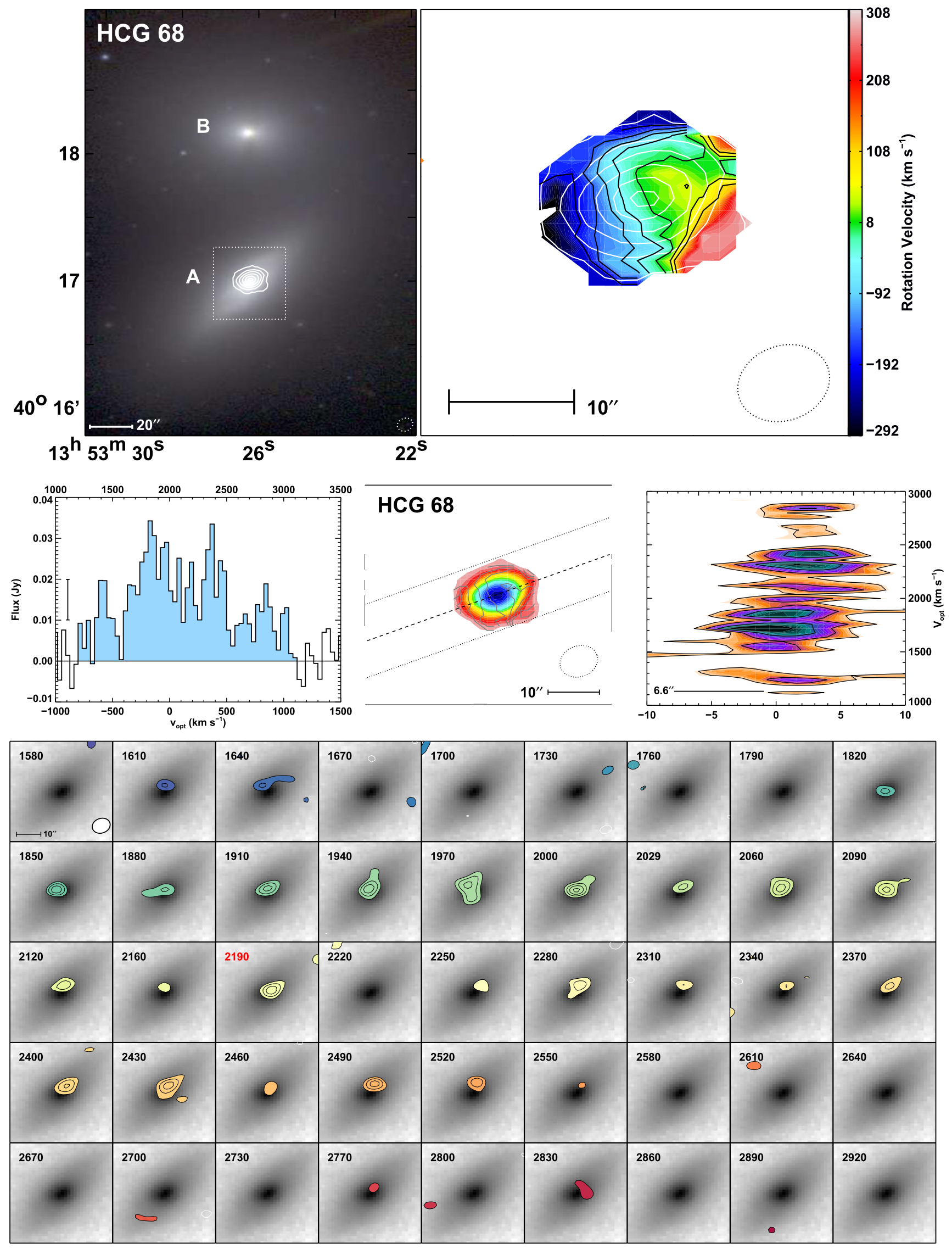

Figure 12. HCG 68. Channel map contours are in $1 \sigma$ steps. 

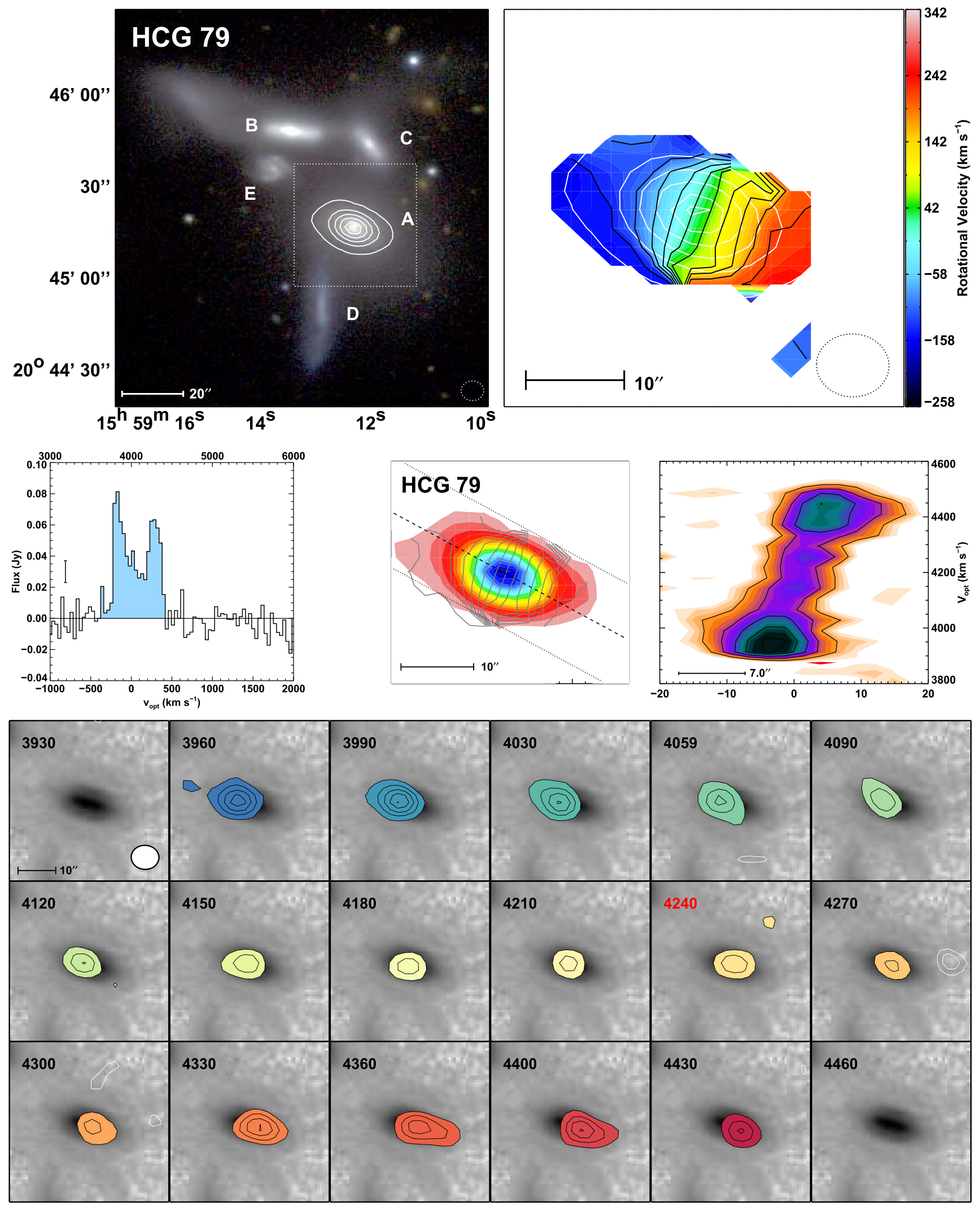

Figure 13. HCG 79. Channel map contours are in $3 \sigma$ steps.

can be found in Alatalo et al. (2014a). The molecular gas in HCG 57d is morphologically classified as a ring.

HCG 68 a (Figure 12) was previously un-detected by the IRAM $30 \mathrm{~m}$ in the ATLAS ${ }^{3 \mathrm{D}}$ survey (Young et al. 2011), but was later detected by Lisenfeld et al. (2014). The CARMA observation helps shed light on why this was the case. The molecular gas in HCG 68a is not only compact (unresolved by the CARMA beam), but also 

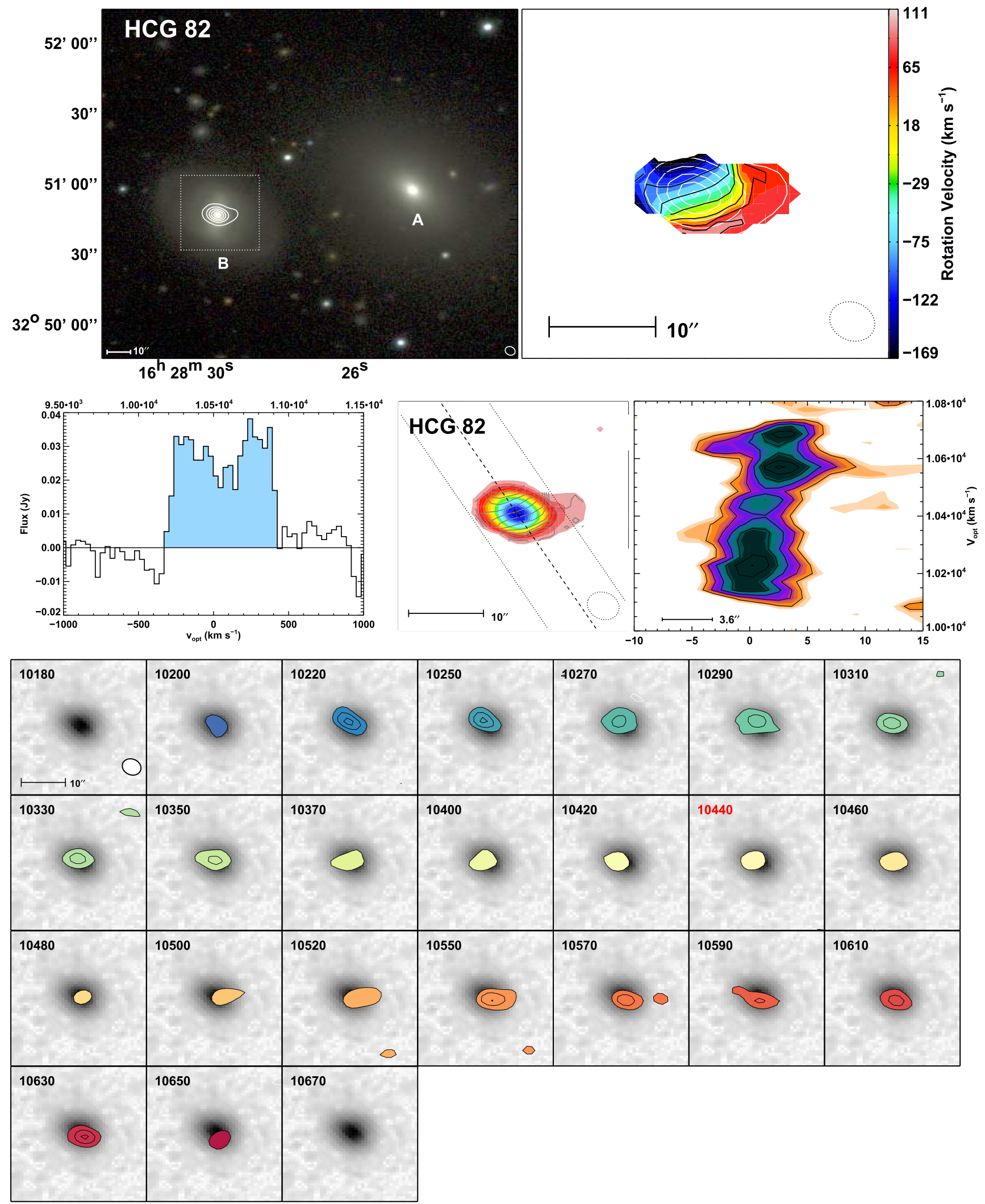

Figure 14. HCG 82. Channel map contours are in $3 \sigma$ steps.

very broad $\left(\Delta v \approx 2000 \mathrm{~km} \mathrm{~s}^{-1}\right)$. HCG 68a also appears to have rotation velocities in its $\mathrm{CO}$ emission that makes it an outlier on the $M_{\mathrm{gal}}-v_{\mathrm{rot}, \mathrm{CO}}$ relation (Davis et al. 2011). HCG 68a and HCG 68b were also both detected in $3 \mathrm{~mm}$ continuum emission. DecompIR (Mullaney et al. 2011) suggests that about $9 \%$ of the far-IR emission (Bitsakis et al. 2014) originates from the AGN in this system. The AGN in HCG 68a has an $2-10 \mathrm{keV}$ X-ray luminosity of 

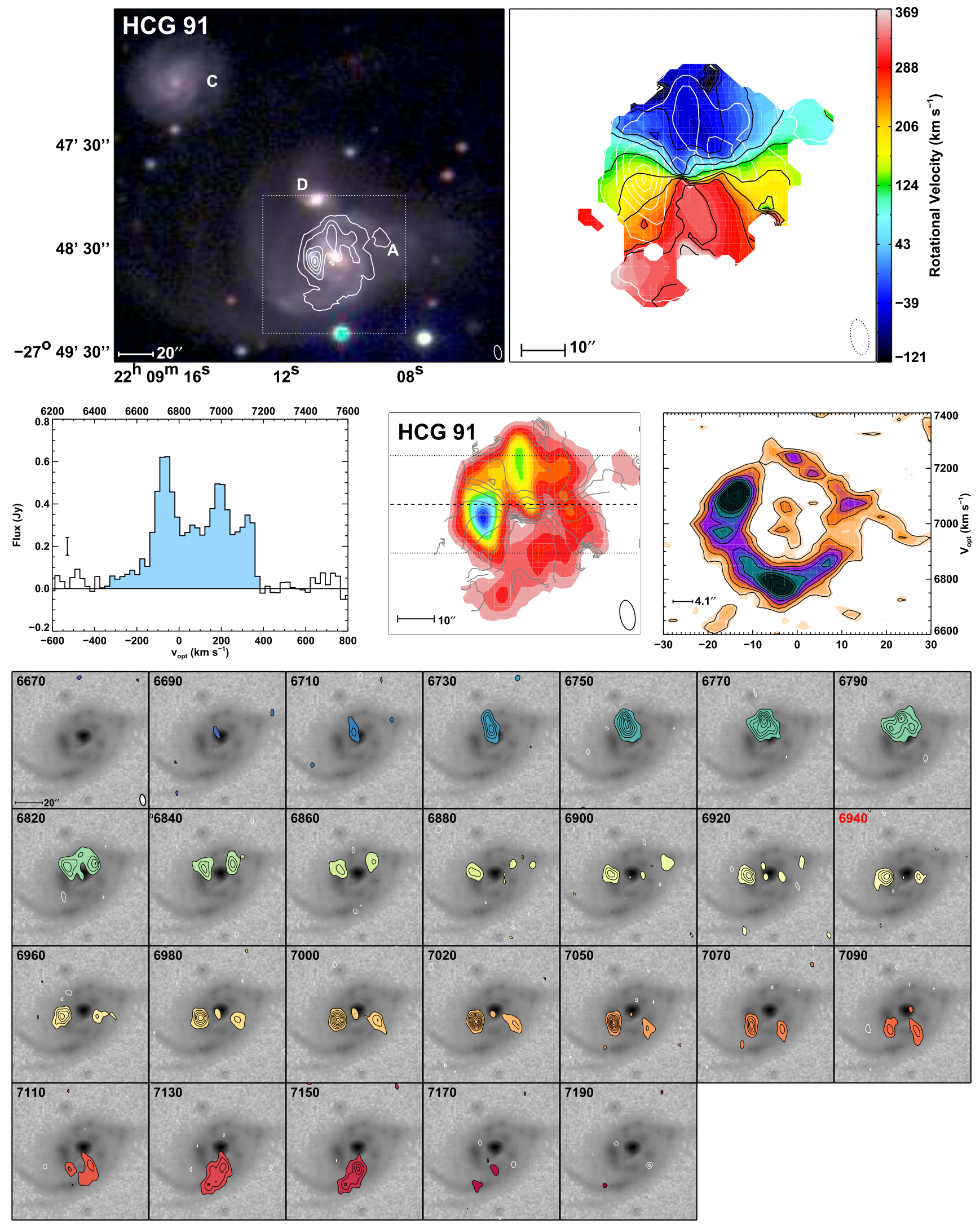

Figure 15. HCG 91. Channel map contours are in $3 \sigma$ steps.

$L_{\mathrm{X}}=1.6 \times 10^{40} \mathrm{erg} \mathrm{s}^{-1}$ (Evans et al. 2010), which could account for the majority of the far-IR emission if the obscuring column of molecular gas is sufficiently high (which the CARMA observations suggest might be the case). HCG 68a is also one of the strongest MOHEGs, with $L_{\mathrm{H}_{2} \text {, warm }} / L_{7.7 \mu \mathrm{mPAH}}=0.741$, and is an outlier in IR color space among the CO-imaged HCG galaxies. HCG 68a has Spitzer colors corresponding to an early-type galaxy, though 

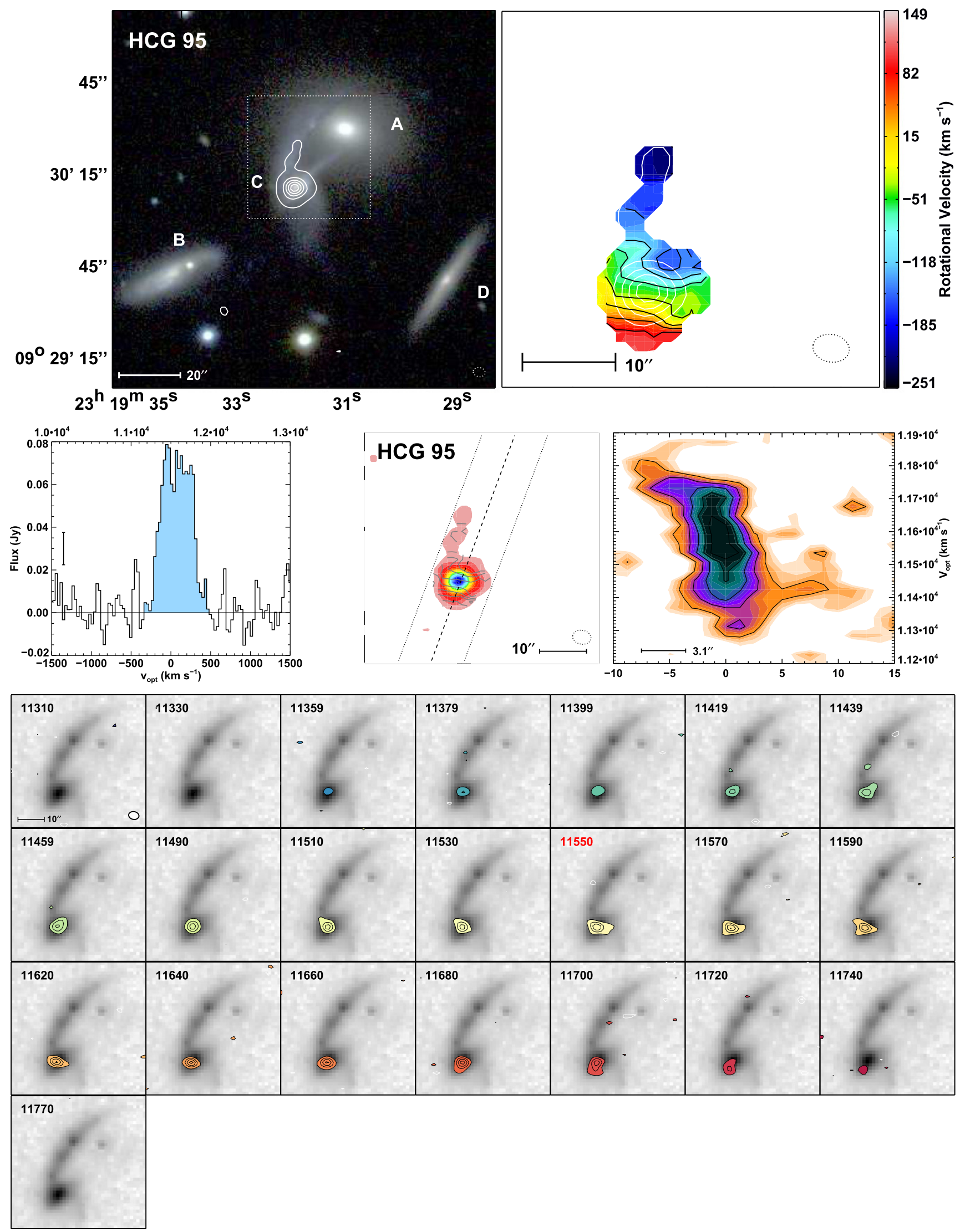

Figure 16. HCG 95. Channel map contours are in $3 \sigma$ steps.

these colors might also be due to a buried AGN with very few intermediate aged stars. HCG 68a is also the only galaxy that is found with WISE colors completely consistent with the elliptical sample (Alatalo et al. 2014b), but sits within the optical green valley. The molecular gas in HCG 68a is morphologically classified as a disk. 

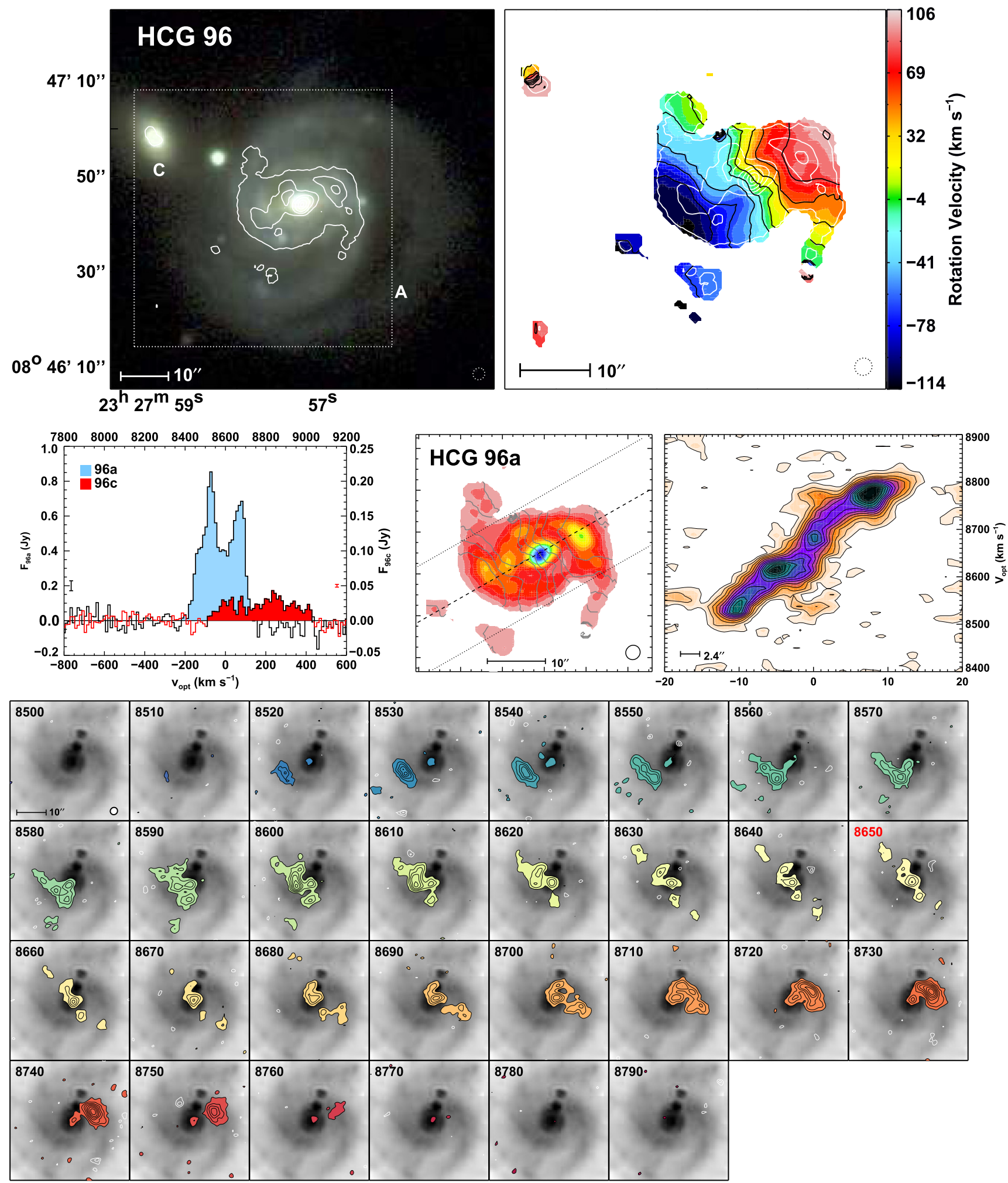

Figure 17. HCG 96. Channel map contours are in $3 \sigma$ steps. Channel maps and a PVD of 96c can be found in Figures 20 and 19 , respectively.

HCG 79a (Figure 13), a member of Seyfert's Sextet (Seyfert 1951), ${ }^{32}$ is a near edge-on red early-type galaxy with a prominent dust lane. HCG 79a is optically classified as having an AGN and is found in the WISE IRTZ. The molecular gas in HCG 79a is morphologically classified as a disk.

\footnotetext{
${ }^{32}$ Although HCG 79c later identified to be a background galaxy.
}

$H C G 82 b$ (Figure 14) is the only CO-imaged HCG that was successfully detected in $3 \mathrm{~mm}$ continuum but not in $1.4 \mathrm{GHz}$ continuum. Despite this, there is an optically identified AGN (Martinez et al. 2010), so the $3 \mathrm{~mm}$ continuum is most likely due to an AGN. HCG 82b is a MOHEG (Cluver et al. 2013) located in the red sequence, as well as the Spitzer gap and the WISE IRTZ. Figure 14 shows HCG 82b contains a stellar bar, 

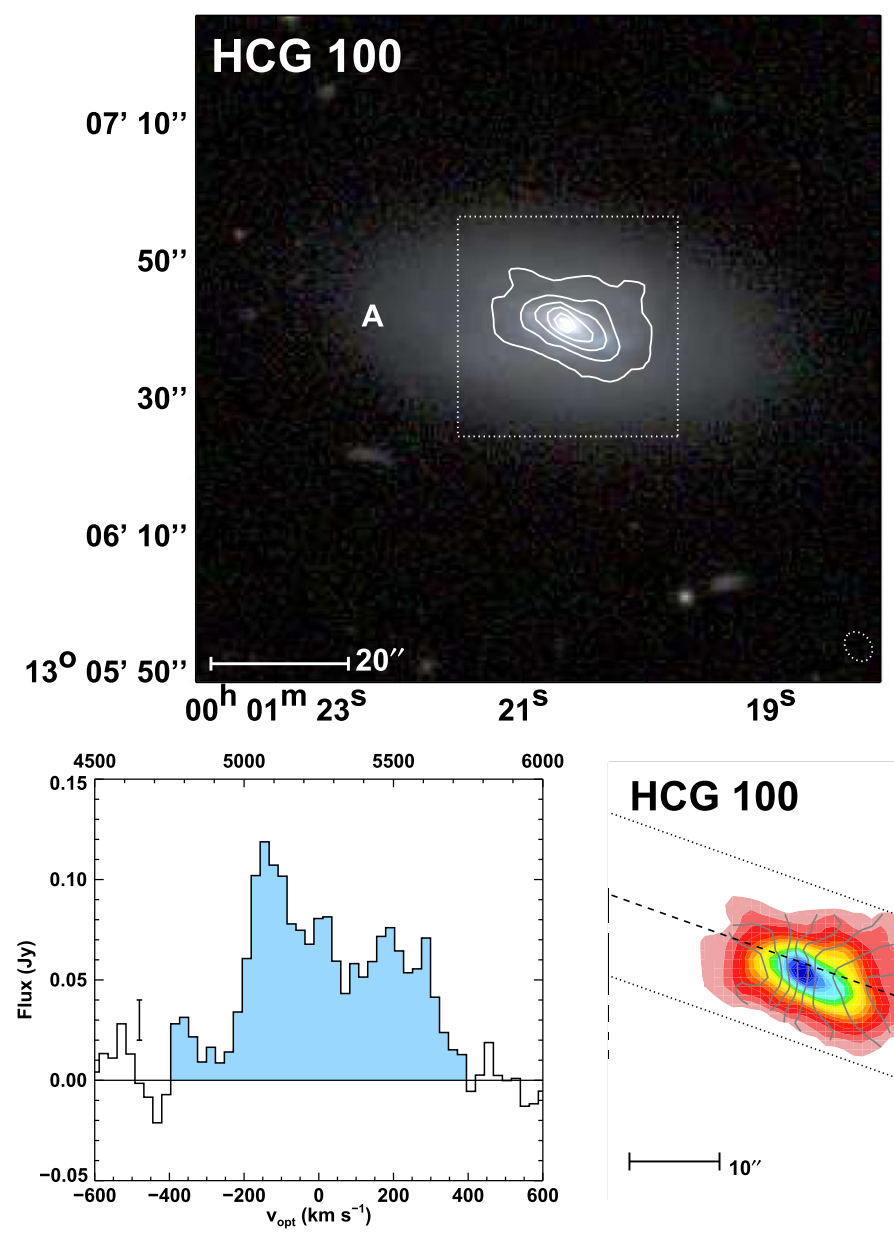
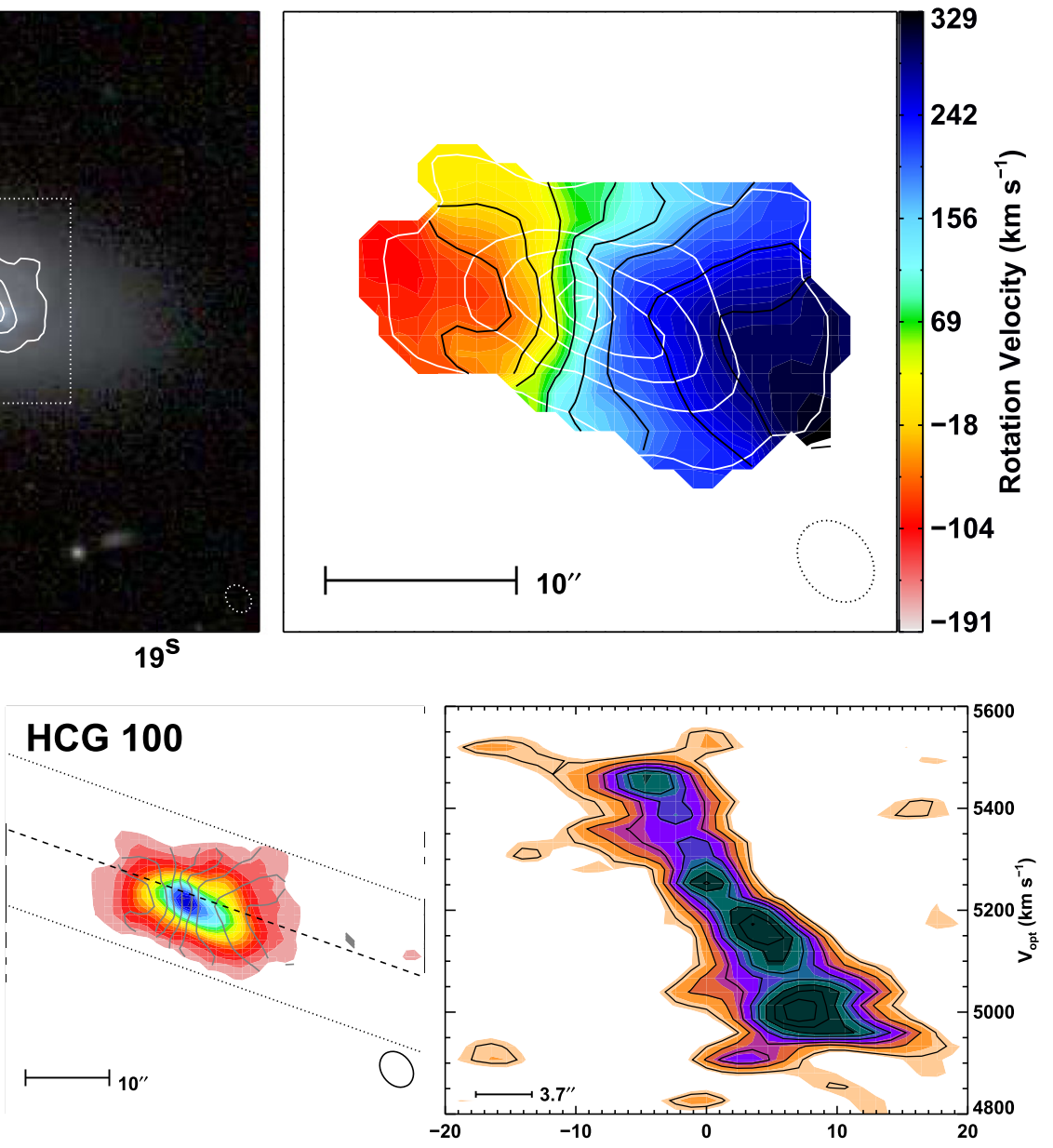

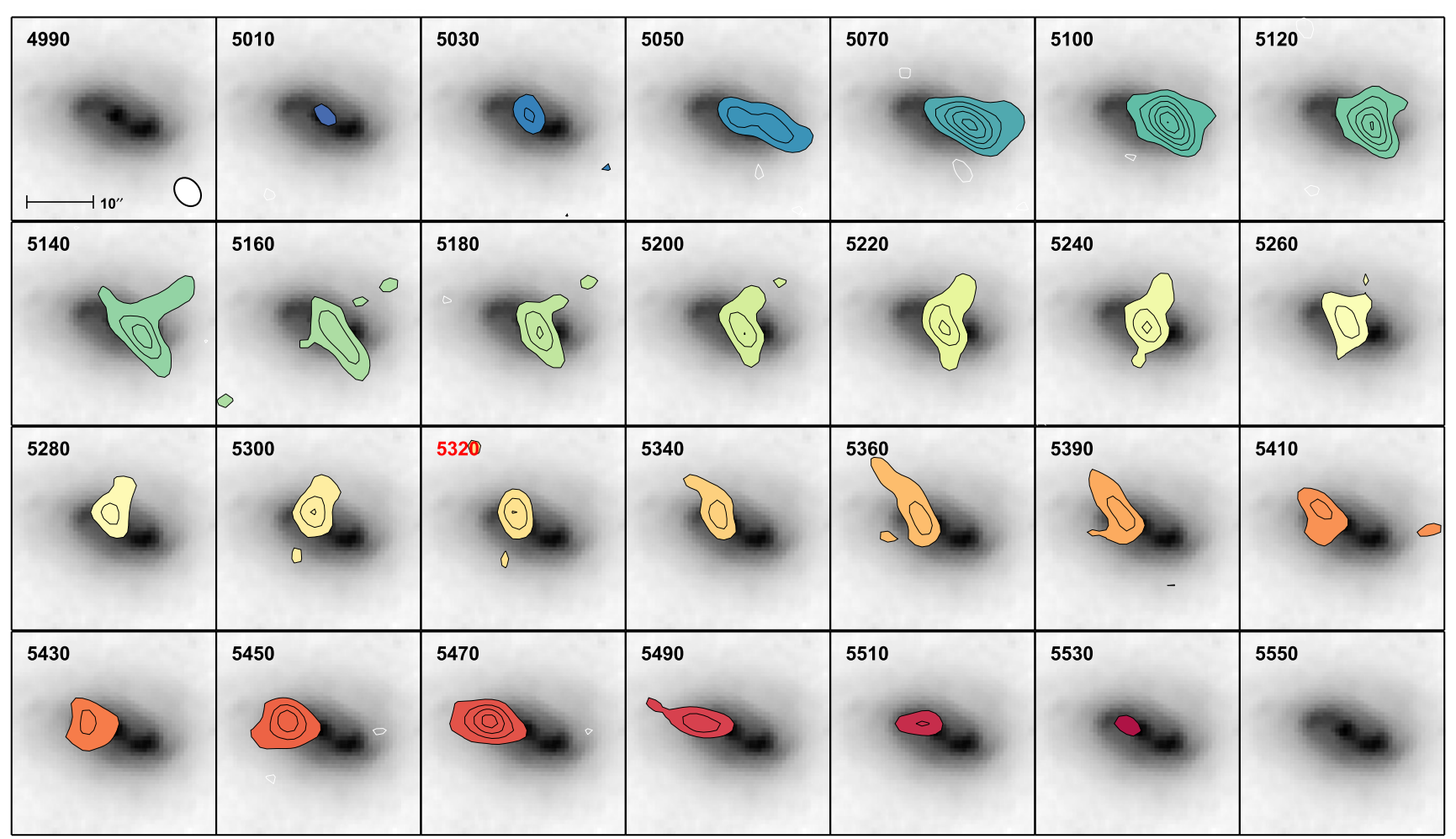

Figure 18. HCG 100. Channel map contours are in $3 \sigma$ steps. 

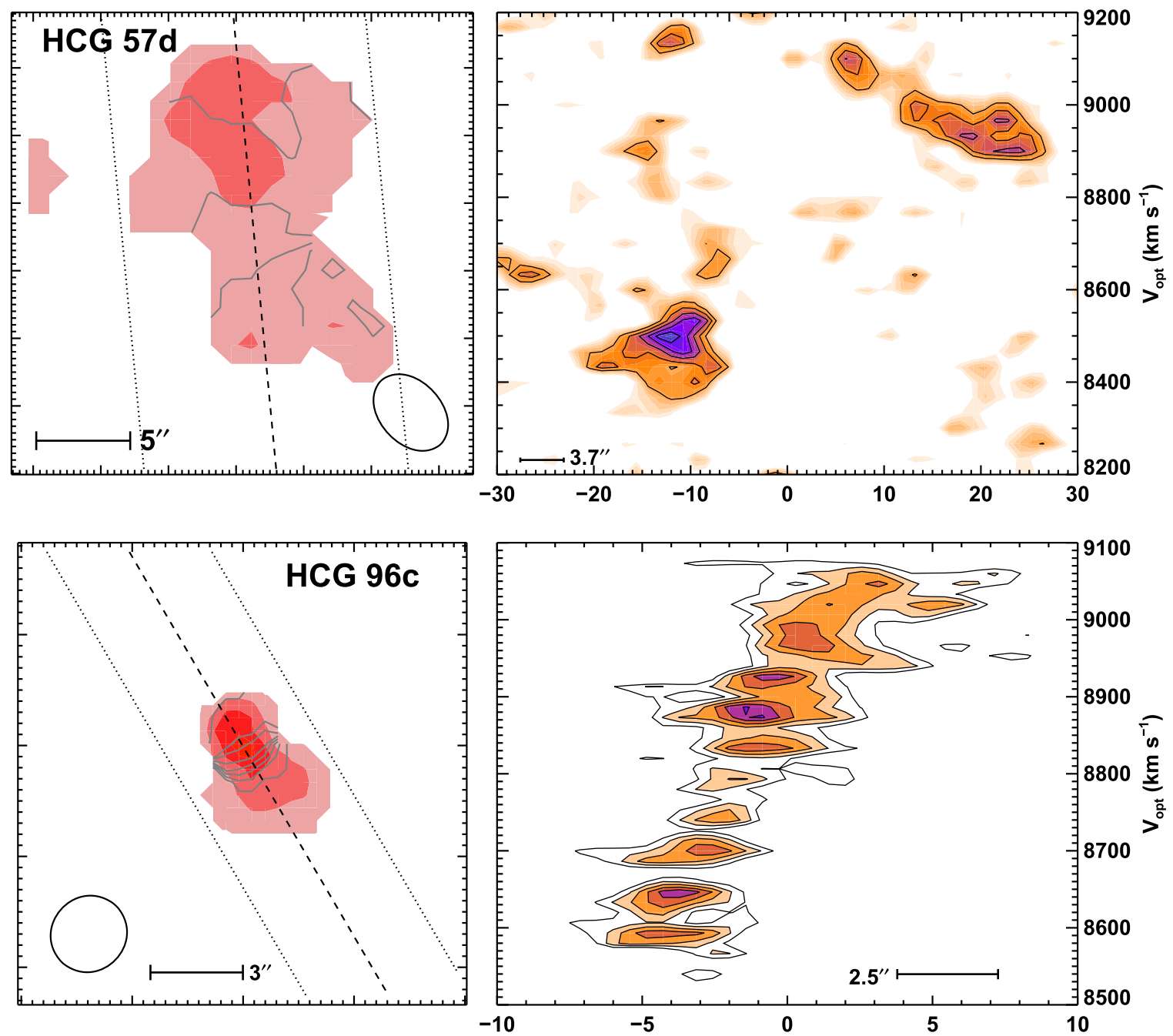

Figure 19. PVDs of HCG 57d (top) and HCG 96c (bottom). The molecular gas in HCG 57d appears to be consistent with a ring (seen in PAH emission in Figure 11 and described in Alatalo et al. 2014a). The molecular gas in HCG 96c seems to be consistent with a rotating disk.

where the molecular gas does not appear aligned with the bar. The lip seen at the edge of the molecular disk seems consistent with a warp, and thus the molecular gas in this galaxy is morphologically classified as mildly disrupted.

$H C G 91 a$ (Figure 15) is a nearly face-on spiral galaxy that is blue in optical and IR colors, with the $\mathrm{CO}(1-0)$ being brightest in the northeast quadrant of the galaxy. Deep $\mathrm{H} \alpha$ imaging from Eigenthaler et al. (2015) seems to indicate that this might be the location of a bow shock. It is the only galaxy in this survey classified as a Seyfert 1 (Cluver et al. 2013). The deep optical imaging of HCG91a also indicates that it is undergoing a significant interaction with HCG 91c (Eigenthaler et al. 2015), showing multiple tidal tails, including one connecting the two interacting galaxies. Vogt et al. (2015) also showed that the CO and optical line velocities were offset from that of the $\mathrm{H}$. HCG 91a has many different kinematic components but appears to have a blueshifted line wing that runs directly north-to-south from the nucleus of the galaxy. DecompIR (Mullaney et al. 2011) suggests that up to $35 \%$ of the far-IR (Bitsakis et al. 2014) from the center is due to an AGN. The molecular gas in HCG 91a is morphologically classified as a spiral.

$H C G 95 c$ (Figure 16) mainly shows regular rotation, but the $\mathrm{CO}(1-0)$ emission extends into the tidal tail between HCG 95a and $95 \mathrm{c}$, which is also seen prominently in the $8 \mu \mathrm{m}$ non-stellar emission in the channel map of Figure 16. Shells are also present in the optical light. Spitzer IRAC colors place this galaxy on the edge of the Spitzer IR gap closer to those of dusty spirals, but its $\mathrm{H}_{2} / \mathrm{PAH}$ ratio indicates that it is a MOHEG (Cluver et al. 2013). Unlike other transition galaxies, this one has a high specific SFR. HCG 95c contains radio emission, and is spectrally classified as an AGN (Martinez et al. 2010). The molecular gas in HCG 95c is morphologically classified as mildly disrupted.

HCG $96 a$ (Figure 17) is the only CO-imaged HCG galaxy that could be identified as containing an AGN solely from its mid-IR spectrum (Cluver et al. 2013), that is also classified as an AGN by optical spectroscopy (Martinez et al. 2010). Deep optical imaging (Eigenthaler et al. 2015) shows that HCG 96a is undergoing an interaction with HCG 96c, with multiple tidal tails present, including stellar light connecting HCG 96a and 96c (Verdes-Montenegro et al. 1997). Optical and WISE IR colors are all consistent with a star-forming galaxy, though this object is located in the AGN wedge in Spitzer colors (Lacy et al. 2004). Figure 17 shows that the molecular gas contains multiple components, including spiral structure, a bar component and a ring. Thus the molecular gas in 


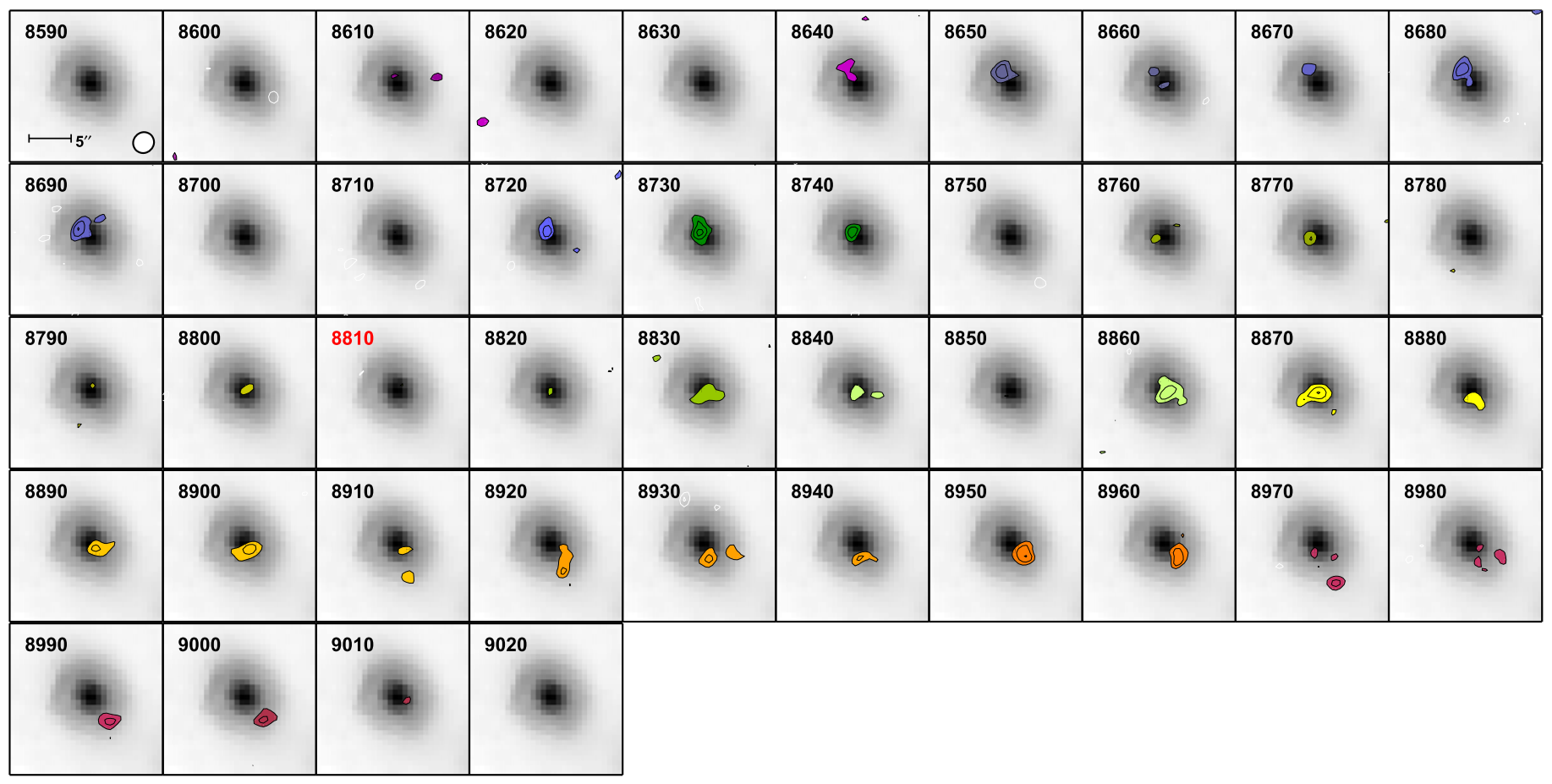

Figure 20. HCG 96c. Channel map contours are in $1 \sigma$ steps.

HCG 96a is morphologically classified as both a spiral and a bar+ring.

HCG 96c (Figure 17) is interacting with HCG 96a, as mentioned above, and appears in the optical green valley, and has Spitzer and WISE colors consistent with star-forming galaxies. The $1.4 \mathrm{GHz}$ emission indicates that HCG 96c might contain a radio-bright AGN. HCG 96c also has a broad $\mathrm{CO}(1-0)$ spectrum, but that is possibly due to its edge-on orientation. The molecular gas in HCG 96c is morphologically classed as a disk.

HCG 100a (Figure 18) exhibits prominent spiral structure, with prolific SF activity in its center (seen in optical light and PAH emission in Figure 18). HCG 100a does not exhibit any outward signs of interaction, such as tidal tails, and is identified as star-forming in both optical and IR colors. HCG 100a is spectrally identified as containing an AGN (Martinez et al. 2010), consistent with its detection in $3 \mathrm{~mm}$ continuum and $1.4 \mathrm{GHz}$ emission. The majority of the molecular gas in HCG 100a seems to be undergoing regular rotation, but there also seems to be a minor-axis component that includes higher velocities (seen as wings in the $\mathrm{CO}(1-0)$ spectrum), possibly due to a bar or an outflow. Despite the presence of a putative minor axis component of the molecular gas in HCG 100a, we morphologically classify this molecular gas as a disk.

\section{APPENDIX C COMPLETE FIGURES FOR EACH HCG}

Figures for each individual HCG are shown in Figures 7-18. For each HCG, we show five figures, as well as an additional figures for HCGs where CARMA detected two galaxies: HCG 57d (Figure 19(a)) and 96c (Figures 19(b) and 20). The outlined box in the image (white dotted line) represents the area of the corresponding moment 1 map. North is up and east is left in all images.
Top left: The $\mathrm{CO}(1-0)$ integrated intensity (moment 0$)$ map (white contours) overlaid on an optical 3-color image, either $g$, $r, i$ from the Sloan Digital Sky Survey (HCG 25, HCG 47, HCG 57, HCG 68, HCG 79, HCG 82, HCG 95, HCG 96, HCG 100), the Digitized Sky Survey (HCG 40, HCG 55), or the Swift archive (HCG 91).

Top right: The $\mathrm{CO}(1-0)$ mean velocity (moment1) map, overlaid with the moment0 map (white contours). The velocities are relative to the systemic velocity of each source.

Middle left: The integrated $\mathrm{CO}(1-0)$ spectrum, which was constructed by summing all pixels in each channel using the moment0 mask as a clip mask.

Middle center and right: The PVD, taken by slicing the $\mathrm{CO}(1-0)$ cube in a plane and summing over a specific region. The moment0 map (middle center) outlines the PVD integration area (dashed black line for the center, dotted gray lines for the boundaries), and the corresponding PVD shows the velocity structure tangent to the velocity slice (middle right).

Bottom: The $\mathrm{CO}(1-0)$ channel maps are overlaid on the Spitzer $8 \mu \mathrm{m}$ nonstellar emission. The Spitzer $8 \mu \mathrm{m}$ nonstellar maps were created by subtracting a scaled $3.6 \mu \mathrm{m}$ map from IRAC from the $8.0 \mu \mathrm{m}$ IRAC map using the scale factor of 0.232 for late-type galaxies (Helou et al. 2004). The shading corresponds to a signal-to-noise ratio of 3 in each channel (except where noted otherwise), with additional contours in either $1 \sigma$ or $3 \sigma$ steps. The panel that corresponds to the systemic velocity has its velocity labeled in red.

\section{REFERENCES}

Aalto, S., Muller, S., Sakamoto, K., et al. 2012, A\&A, 546, A68 Abazajian, K. N., Adelman-McCarthy, J. K. \& the SDSS Collaboration et al. 2009, ApJS, 182, 543

Alatalo, K. 2015, ApJL, 801, L17

Alatalo, K., Appleton, P. N., Lisenfeld, U., et al. 2014a, ApJ, 795, 159

Alatalo, K., Blitz, L., Young, L. M., et al. 2011, ApJ, 735, 88 
Alatalo, K., Cales, S. L., Appleton, P. N., et al. 2014b, ApJL, 794, L13 Alatalo, K., Crocker, A. F., Aalto, S., et al. 2015a, MNRAS, 450, 3874 Alatalo, K., Davis, T. A., Bureau, M., et al. 2013, MNRAS, 432, 1796 Alatalo, K., Lacy, M., Lanz, L., et al. 2015b, ApJ, 798, 31

Alatalo, K., Nyland, K., Graves, G., et al. 2014c, ApJ, 780, 186 Allen, M. G., Groves, B. A., Dopita, M. A., et al. 2008, ApJS, 178, 20 Appleton, P. N., Xu, K. C., Reach, W., et al. 2006, ApJL, 639, L51 Athanassoula, E. 1996, in ASP Conf. Ser., IAU Coll. 157, Barred Galaxies, Vol. 91, ed. R. Buta, D. A. Crocker \& B. G. Elmegreen (San Francisco, CA: ASP), 309

Athanassoula, E., \& Bureau, M. 1999, ApJ, 522, 699

Baldry, I. K., Glazebrook, K., Brinkmann, J., et al. 2004, ApJ, 600, 681

Becker, R. H., White, R. L., \& Helfand, D. J. 1995, ApJ, 450, 559

Bekki, K., Couch, W. J., \& Shioya, Y. 2002, ApJ, 577, 651

Bell, E. F., McIntosh, D. H., Katz, N., \& Weinberg, M. D. 2003, ApJS, 149, 289

Best, P. N., Kauffmann, G., Heckman, T. M., et al. 2005, MNRAS, 362, 25 Bigiel, F., Leroy, A., Walter, F., et al. 2008, AJ, 136, 2846

Bitsakis, T., Charmandaris, V., Appleton, P. N., et al. 2014, A\&A, 565, A25 Bitsakis, T., Charmandaris, V., da Cunha, E., et al. 2011, A\&A, 533, A142

Bitsakis, T., Charmandaris, V., Le Floc'h, E., et al. 2010, A\&A, 517, A75

Blanton, M. R., \& Moustakas, J. 2009, ARA\&A, 47, 159

Bock, D. C.-J., Bolatto, A. D., Hawkins, D. W., et al. 2006, Proc. SPIE, 6267, 626713

Bolatto, A. D., Leroy, A. K., Rosolowsky, E., Walter, F., \& Blitz, L. 2008, ApJ, 686, 948

Bolatto, A. D., Wolfire, M., \& Leroy, A. K. 2013, ARA\&A, 51, 207

Borthakur, S., Yun, M. S., \& Verdes-Montenegro, L. 2010, ApJ, 710, 385

Brown, M. J. I., Jannuzi, B. T., Floyd, D. J. E., \& Mould, J. R. 2011, ApJL, 731, L41

Bryant, P. M., \& Scoville, N. Z. 1999, AJ, 117, 2632

Burton, W. B. 1971, A\&A, 10, 76

Cales, S. L., Alatalo, K., Rich, J. A., et al. 2015, ApJS, submitted

Calzetti, D., Kennicutt, R. C., Engelbracht, C. W., et al. 2007, ApJ, 666, 870

Chang, Y.-Y., van der Wel, A., da Cunha, E., \& Rix, H.-W. 2015, ApJS, 219, 8

Chung, A., Narayanan, G., Yun, M. S., Heyer, M., \& Erickson, N. R. 2009, AJ, 138,858

Cicone, C., Feruglio, C., Maiolino, R., et al. 2012, A\&A, 543, A99

Cicone, C., Maiolino, R., Sturm, E., et al. 2014, A\&A, 562, A21

Ciesla, L., Charmandaris, V., Georgakakis, A., et al. 2015, A\&A, 576, A10

Cluver, M. E., Appleton, P. N., Ogle, P., et al. 2013, ApJ, 765, 93

Condon, J. J. 1992, ARA\&A, 30, 575

Condon, J. J., Cotton, W. D., Greisen, E. W., et al. 1998, AJ, 115, 1693

Cresci, G., Hicks, E. K. S., Genzel, R., et al. 2009, ApJ, 697, 115

Crocker, A. F., Bureau, M., Young, L. M., \& Combes, F. 2011, MNRAS, 410, 1197

da Cunha, E., Charlot, S., \& Elbaz, D. 2008, MNRAS, 388, 1595

da Cunha, E., Eminian, C., Charlot, S., et al. 2010, MNRAS, 403, 1894

Dale, D. A., Cohen, S. A., Johnson, L. C., et al. 2009, ApJ, 703, 517

Davis, T. A., Bureau, M., Young, L. M., et al. 2011, MNRAS, 414, 968

Davis, T. A., Young, L. M., Crocker, A. F., et al. 2014, MNRAS, 444, 3427

Dib, S., Bell, E., \& Burkert, A. 2006, ApJ, 638, 797

Donoso, E., Yan, L., Tsai, C., et al. 2012, ApJ, 748, 80

Downes, D., \& Solomon, P. M. 1998, ApJ, 507, 615

Eigenthaler, P., Ploeckinger, S., Verdugo, M., \& Ziegler, B. 2015, MNRAS, 451, 2793

Eliche-Moral, M. C., González-García, A. C., Aguerri, J. A. L., et al. 2012, A\&A, 547, A48

Evans, I. N., Primini, F. A., Glotfelty, K. J., et al. 2010, ApJS, 189, 37

Fazio, G. G., Hora, J. L., Allen, L. E., et al. 2004, ApJS, 154, 10

Feruglio, C., Maiolino, R., Piconcelli, E., et al. 2010, A\&A, 518, L155

Fischer, J., Sturm, E., González-Alfonso, E., et al. 2010, A\&A, 518, L41

Fisher, D. B., Bolatto, A., Drory, N., et al. 2013, ApJ, 764, 174

French, K. D., Yang, Y., Zabludoff, A., et al. 2015, ApJ, 801, 1

García-Burillo, S., Combes, F., Usero, A., et al. 2014, A\&A, 567, A125

Garcia-Burillo, S., Combes, F., Usero, A., et al. 2015, A\&A, 580, 35

Genzel, R., Tacconi, L. J., Gracia-Carpio, J., et al. 2010, MNRAS, 407, 2091

Guillard, P., Boulanger, F., Lehnert, M. D., et al. 2015, A\&A, 574, A32

Guillard, P., Boulanger, F., Pineau Des Forêts, G., \& Appleton, P. N. 2009, A\&A, 502, 515

Guillard, P., Boulanger, F., Pineau des Forêts, G., et al. 2012a, ApJ, 749, 158

Guillard, P., Ogle, P. M., Emonts, B. H. C., et al. 2012b, ApJ, 747, 95

Helfer, T. T., Thornley, M. D., Regan, M. W., et al. 2003, ApJS, 145, 259

Helou, G., Roussel, H., Appleton, P., et al. 2004, ApJS, 154, 253

Hickson, P. 1982, ApJ, 255, 382

Hickson, P. 1997, ARA\&A, 35, 357
Hickson, P., Mendes de Oliveira, C., Huchra, J. P., \& Palumbo, G. G. 1992, ApJ, 399, 353

Hopkins, P. F., Hernquist, L., Cox, T. J., \& Kereš, D. 2008, ApJS, 175, 356 Hopkins, P. F., Hernquist, L., Cox, T. J., et al. 2006, ApJS, 163, 1

Houck, J. R., Roellig, T. L., van Cleve, J., et al. 2004, ApJS, 154, 18 Iono, D., Wilson, C. D., Yun, M. S., et al. 2009, ApJ, 695, 1537

Johnson, K. E., Hibbard, J. E., Gallagher, S. C., et al. 2007, AJ, 134, 1522

Kauffmann, J., Pillai, T., \& Zhang, Q. 2013, ApJL, 765, L35

Kennicutt, R. C., Jr. 1998, ApJ, 498, 541

Ko, J., Hwang, H. S., Lee, J. C., \& Sohn, Y.-J. 2013, ApJ, 767, 90

Konstantopoulos, I. S., Appleton, P. N., Guillard, P., et al. 2014, ApJ, 784, 1

Krumholz, M. R., Dekel, A., \& McKee, C. F. 2012, ApJ, 745, 69

Lacy, M., Petric, A. O., Sajina, A., et al. 2007, AJ, 133, 186

Lacy, M., Ridgway, S. E., Gates, E. L., et al. 2013, ApJS, 208, 24

Lacy, M., Storrie-Lombardi, L. J., Sajina, A., et al. 2004, ApJS, 154, 166

Lanz, L., Hayward, C. C., Zezas, A., et al. 2014, ApJ, 785, 39

Lanz, L., Ogle, P. M., Evans, D., et al. 2015, ApJ, 801, 17

Lanz, L., Zezas, A., Brassington, N., et al. 2013, ApJ, 768, 90

Leon, S., Combes, F., \& Menon, T. K. 1998, A\&A, 330, 37

Lintott, C. J., Schawinski, K., Slosar, A., et al. 2008, MNRAS, 389, 1179

Lisenfeld, U., Appleton, P. N., Cluver, M. E., et al. 2014, A\&A, 570, A24

Lisenfeld, U., Espada, D., Verdes-Montenegro, L., et al. 2011, A\&A, 534, A102

Liu, J. 2011, ApJS, 192, 10

Longmore, S. N., Rathborne, J., Bastian, N., et al. 2012, ApJ, 746, 117

Makarov, D., Prugniel, P., Terekhova, N., Courtois, H., \& Vauglin, I. 2014, A\&A, 570, A13

Malhotra, S. 1995, ApJ, 448, 138

Martig, M., Bournaud, F., Teyssier, R., \& Dekel, A. 2009, ApJ, 707, 250

Martig, M., Crocker, A. F., Bournaud, F., et al. 2013, MNRAS, 432, 1914

Martinez, M. A., Del Olmo, A., Coziol, R., \& Perea, J. 2010, AJ, 139, 1199

Martinez-Badenes, V., Lisenfeld, U., Espada, D., et al. 2012, A\&A, 540, A96

Menon, T. K., \& Hickson, P. 1985, ApJ, 296, 60

Mullaney, J. R., Alexander, D. M., Goulding, A. D., \& Hickox, R. C. 2011, MNRAS, 414, 1082

Nesvadba, N. P. H., Boulanger, F., Salomé, P., et al. 2010, A\&A, 521, A65

Nyland, K., Young, L. M., Wrobel, J. M., et al. 2015, MNRAS, submitted

Ogle, P., Antonucci, R., Appleton, P. N., \& Whysong, D. 2007, ApJ, 668, 699

Ogle, P., Boulanger, F., Guillard, P., et al. 2010, ApJ, 724, 1193

Ogle, P., Whysong, D., \& Antonucci, R. 2006, ApJ, 647, 161

Ogle, P. M., Lanz, L., \& Appleton, P. N. 2014, ApJL, 788, L33

O’Sullivan, E., Vrtilek, J. M., David, L. P., et al. 2014a, ApJ, 793, 74

O’Sullivan, E., Zezas, A., Vrtilek, J. M., et al. 2014b, ApJ, 793, 73

Palouš, J. 2005, RvMA, 18, 125

Peterson, B. W., Appleton, P. N., Helou, G., et al. 2012, ApJ, 751, 11

Plauchu-Frayn, I., Del Olmo, A., Coziol, R., \& Torres-Papaqui, J. P. 2012, A\&A, 546, A48

Ponman, T. J., Bourner, P. D. J., Ebeling, H., \& Böhringer, H. 1996, MNRAS, 283, 690

Privon, G. C., Barnes, J. E., Evans, A. S., et al. 2013, ApJ, 771, 120

Qu, Y., Di Matteo, P., Lehnert, M., van Driel, W., \& Jog, C. J. 2010, A\&A, 515, A11

Rasmussen, J., Ponman, T. J., Verdes-Montenegro, L., Yun, M. S., \& Borthakur, S. 2008, MNRAS, 388, 1245

Rémy-Ruyer, A., Madden, S. C., Galliano, F., et al. 2014, A\&A, 563, A31

Renaud, F., Boily, C. M., Naab, T., \& Theis, C. 2009, ApJ, 706, 67

Renaud, F., Bournaud, F., Kraljic, K., \& Duc, P.-A. 2014, MNRAS, 442, L33

Rich, J. A., Kewley, L. J., \& Dopita, M. A. 2011, ApJ, 734, 87

Rowlands, K., Wild, V., Nesvadba, N., et al. 2015, MNRAS, 448, 258

Saintonge, A., Kauffmann, G., Kramer, C., et al. 2011a, MNRAS, 415, 32

Saintonge, A., Kauffmann, G., Wang, J., et al. 2011b, MNRAS, 415, 61

Salpeter, E. E. 1955, ApJ, 121, 161

Sanders, D. B., \& Mirabel, I. F. 1996, ARA\&A, 34, 749

Sanders, D. B., Scoville, N. Z., \& Soifer, B. T. 1991, ApJ, 370, 158

Sandstrom, K. M., Leroy, A. K., Walter, F., et al. 2013, ApJ, 777, 5

Sargent, W. L. W. 1968, ApJL, 153, L135

Sault, R. J., Teuben, P. J., \& Wright, M. C. H. 1995, in ASP Conf. Ser. 77, Astronomical Data Analysis Software and Systems IV, ed. R. A. Shaw, H. E. Payne \& J. J. E. Hayes (San Francisco, CA: ASP), 433

Scalo, J., \& Elmegreen, B. G. 2004, ARA\&A, 42, 275

Schawinski, K., Urry, C. M., Simmons, B. D., et al. 2014, MNRAS, 440, 889

Serra, P., Oosterloo, T., Morganti, R., et al. 2012, MNRAS, 422, 1835

Seyfert, C. K. 1951, PASP, 63, 72

Skrutskie, M. F., Cutri, R. M., Stiening, R., et al. 2006, AJ, 131, 1163

Smethurst, R. J., Lintott, C. J., Simmons, B. D., et al. 2015, MNRAS, 450, 435

Spergel, D. N., Bean, R., Doré, O., et al. 2007, ApJS, 170, 377 
Springel, V., Di Matteo, T., \& Hernquist, L. 2005, ApJL, 620, L79

Stern, J., \& Laor, A. 2012, MNRAS, 423, 600

Strateva, I., Ivezić, Ž, Knapp, G. R., et al. 2001, AJ, 122, 1861

Struck, C. 1999, PhR, 321, 1

Sturm, E., González-Alfonso, E., Veilleux, S., et al. 2011, ApJL, 733, L16

Tinsley, B. M. 1978, ApJ, 222, 14

Toomre, A. 1964, ApJ, 139, 1217

Toomre, A., \& Toomre, J. 1972, ApJ, 178, 623

U, V., Sanders, D. B., Mazzarella, J. M., et al. 2012, ApJS, 203, 9

Ueda, J., Iono, D., Yun, M. S., et al. 2014, ApJS, 214, 1

Utomo, D., Blitz, L., Davis, T., et al. 2015, ApJ, 803, 16

Verdes-Montenegro, L., del Olmo, A., Perea, J., et al. 1997, A\&A, 321, 409

Verdes-Montenegro, L., Yun, M. S., Williams, B. A., et al. 2001, A\&A, 377,812
Vogt, F. P. A., Dopita, M. A., Borthakur, S., et al. 2015, MNRAS, 450, 2593

Walker, L. M., Johnson, K. E., Gallagher, S. C., et al. 2010, AJ, 140, 1254

Walter, F., Brinks, E., de Blok, W. J. G., et al. 2008, AJ, 136, 2563

Wilson, C. D., Petitpas, G. R., Iono, D., et al. 2008, ApJS, 178, 189

Wright, E. L., Eisenhardt, P. R. M., Mainzer, A. K., et al. 2010, AJ, 140,1868

Wuyts, S., Förster Schreiber, N. M., van der Wel, A., et al. 2011, ApJ, 742, 96

Yesuf, H. M., Faber, S. M., Trump, J. R., et al. 2014, ApJ, 792, 84

York, D. G., Adelman, J., Anderson, J. E., Jr., et al. 2000, AJ, 120, 1579

Young, L. M., Bureau, M., \& Cappellari, M. 2008, ApJ, 676, 317

Young, L. M., Bureau, M., Davis, T. A., et al. 2011, MNRAS, 414, 940

Yusef-Zadeh, F., Hewitt, J. W., Arendt, R. G., et al. 2009, ApJ, 702, 178

Zabludoff, A. I., \& Mulchaey, J. S. 1998, ApJ, 496, 39 